TITLE:

\title{
Three-dimensional seismic response of a cylindrical tunnel with voids behind the lining
}

$\operatorname{AUTHOR}(\mathrm{S})$ :

Naotoshi, Yasuda; Kazuhiko, Tsukada; Toshihiro, Asakura

\section{CITATION:}

Naotoshi, Yasuda ...[et al]. Three-dimensional seismic response of a cylindrical tunnel with voids behind the lining. Tunnelling and Underground Space Technology 2019, 84: 399-412

ISSUE DATE:

2019-02

URL:

http://hdl.handle.net/2433/243224

\section{RIGHT:}

(c) 2019. This manuscript version is made available under the CC-BY-NC-ND 4.0 license

http://creativecommons.org/licenses/by-nc-nd/4.0/:; The full-text file will be made open to the public on 1 February 2021 in accordance with publisher's 'Terms and Conditions for Self-Archiving'.; This is not the published version. Please cite only the published version.; この論文は出版社版でありません。引用の際には出版社版をご確認ご利用ください。 


\title{
Three-dimensional seismic response of a cylindrical tunnel with voids behind the lining
}

\author{
Naotoshi Yasuda ${ }^{\mathrm{a}, *}, K$ Kazuhiko Tsukada ${ }^{\mathrm{a}}$, Toshihiro Asakurab \\ ${ }^{a}$ Department of Civil and Earth Resources Engineering, Kyoto University, Katsura, \\ Nishigyou-ku, Kyoto 615-8540, Japan \\ ${ }^{b}$ Research group for tunnel engineering, Kajichō, Chiyoda-ku, Tokyo 101-0044, Japan
}

\begin{abstract}
Past experiences in Japan have suggested a high correlation between the presence of voids behind the tunnel lining and seismic damage of tunnels. To confirm this correlation from a theoretical perspective, this study derives three-dimensional elastic solutions for a deep cylindrical tunnel with a void subjected to obliquely incident seismic waves. The three-dimensional seismic response of the tunnel and the effect of voids on the response are investigated. Herein, a void is treated as a partial non-contact boundary between the lining and the ground, with negligible volume. The substructure method and the point-matching method are used to derive the solutions. Numerical results show that the presence of a void does not lead to a large stress concentration on the lining under an oblique-incidence of seismic waves. However, high correlation between large stress concentration on the lining caused by voids before an earthquake and seismic damage of tunnels is suggested. Based on this finding, reinforcement of the lining where large stress concentration has
\end{abstract}

\footnotetext{
*corresponding author. Tel.:+81 75383 7485; Fax: +81 753833218

Email address: yasuda.naotoshi.3x@kyoto-u.ac.jp (Naotoshi Yasuda)
} 
already occurred is crucial for preventing seismic damage of tunnels. Keywords: Tunnel, Void behind the lining, Elastodynamics, Seismic response, Three-dimensional analysis

\section{Introduction}

Compared to surface structures, tunnels are relatively less vulnerable to earthquakes. Some mountain tunnels, however, have experienced significant damage in recent earthquakes including the 1995 Kobe (Asakura and Sato, 1996), the 1999 Chi-Chi (Wang et al., 2001; Chen et al., 2002), the 2004 Niigata (Yashiro et al., 2007), the 2008 Wenchua (Tianbin, 2008; Li, 2012), and the Kumamoto (Zhang et al., 2018). Therefore, understanding the earthquake damage mechanisms of tunnels is crucial.

Mountain tunnels are generally vulnerable to damage when they are subjected a massive earthquake, they are located near an earthquake fault, and they are under certain special conditions (Yashiro et al., 2007). The special conditions include a shallow tunnel, poor geological conditions, sliding of an earthquake fault along which the tunnel is located, and structural defects in the lining. Severely damaged tunnels have been found to meet at least one of these conditions. In old tunnels that were constructed using conventional method (tunnelling method before New Austrian tunnelling method) in Japan, partial discontinuities, or voids, between the lining and the ground are often presented, which adversely affect the lining (Meguid and Dang, 2009; Wang et al., 2014; Meguid and Kamel, 2014). The presence of such voids is one of the most significant factors that cause seismic damage to tunnels, as evidenced by past experiences in Japan. 
The Uonuma tunnel, which was the most severely damaged tunnel in the 2004 Niigata earthquake, has voids behind the lining. The concrete lining of the most severely damaged section had broken off and fallen onto the track with the largest concrete block being approximately $2 \mathrm{~m}^{3}$ and weighing five tons (Yashiro et al., 2007). This damage was considered to be caused by high longitudinal thrust because cracks in the transverse direction of the tunnel and ring cracks were predominant. This damage can not be explained by conventional two-dimensional analysis using the tunnel cross-section (Amorosi and Boldini, 2009; Park et al., 2009). A three-dimensional analysis is necessary to understand the earthquake damage mechanisms.

This paper presents three-dimensional elastic solutions for a deep cylindrical tunnel that consists of a void behind the lining and is subjected to obliquely incident seismic waves. The solutions are derived using the substructure method (Wolf, 1985) and the point-matching method (Yasuda et al., 2017). The three-dimensional seismic response of the tunnel and the effects of a void on the response are investigated.

\section{Theory}

\subsection{Problem definition}

Consider an infinite cylindrical tunnel consisting of a void behind the lining and subjected to a plane harmonic seismic wave propagating at an angle $\phi$ with respect to the axis of the cylindrical tunnel, as shown in Fig. C.1. The surrounding ground is considered to be an infinite elastic, homogeneous, isotropic medium. The lining is treated as an elastic, homogeneous, isotropic medium with an outer radius $R$ and a thickness $h$. 
Because of the azimuthal symmetry of the circular cylinder, an incident pressure wave (P-wave) or an incident shear wave (S-wave) can be assumed to be propagated in the direction of the wavenumber vector $\boldsymbol{k}_{(1)}^{\text {inc }}$ on the $x-z$ plane; here, superscript "inc" denotes an incident wave. The displacement vector of the P-wave $\boldsymbol{u}_{\mathrm{p}(1)}^{\mathrm{inc}}$ is parallel to $\boldsymbol{k}_{(1)}^{\mathrm{inc}}$ and that of the S-wave $\boldsymbol{u}_{\mathrm{s}(1)}^{\text {inc }}$ is on the plane perpendicular to $\boldsymbol{k}_{(1)}^{\mathrm{inc}}$. $\quad \boldsymbol{u}_{\mathrm{s}(1)}^{\mathrm{inc}}$ can be decomposed into two independent vectors: an $\mathrm{S}_{1}$-wave vector $\boldsymbol{u}_{\mathrm{s}_{1}(1)}^{\mathrm{inc}}$, which is parallel to the $y$ axis, and an $\mathrm{S}_{2}$-wave vector $\boldsymbol{u}_{\mathrm{s}_{2}(1)}^{\text {inc }}$, which is on $x-z$ plane. Subscripts (1) and (2) denote the ground and the lining, respectively.

The void is treated as a partially non-contact boundary between the lining and the ground, with a negligible volume. Its circumferential angle is $\theta_{v}$ and the longitudinal length is $L_{v z}$. To derive the theoretical solutions, a periodic structure is assumed in the longitudinal direction, with length $L_{z}$, which is long enough compared to the longitudinal void length $L_{v z} . L_{z}$ equals the longitudinal wavelength and is expressed by the wavelength of an incident wave in the ground $L_{(1)}$ and incident angle $\phi$, as follows:

$$
L_{z}=\frac{L_{(1)}}{\cos \phi}
$$

$L_{(1)}$ equals the $\mathrm{P}$-wave wavelength $L_{p(1)}$ under $\mathrm{P}$-wave incidence and the S-wave wavelength $L_{s(1)}$ under S-wave incidence.

\subsection{Solution scheme}

To evaluate the displacement and stress under the ground-lining interaction, two substructures, namely, the ground and the lining systems, are considered, as shown in Fig. C.2. In this paper, a positive compression is assumed. 
When the ground-lining system is subjected to an incident seismic wave, the resultant displacement of the ground $\boldsymbol{u}_{(1)}$ can be decomposed into two parts:

$$
\boldsymbol{u}_{(1)}=\boldsymbol{u}_{(1)}^{i}+\boldsymbol{u}_{(1)}^{r},
$$

where $\boldsymbol{u}_{(1)}^{i}$ is the primary displacement of the ground with no lining caused by the incident seismic wave and $\boldsymbol{u}_{(1)}^{r}$ is the secondary displacement of the ground with a lining caused by the reflected wave at the interface $r=R$. The secondary displacement can also be interpreted as the displacement caused by the surface loading $\boldsymbol{f}_{(1)}^{r}$ from the ground-lining interface. Superscripts $i$ and $r$ denote initial and reflection, respectively. The resultant surface loading at the ground-lining interface $\boldsymbol{f}_{(1)}$ is equivalent to $\boldsymbol{f}_{(1)}^{r}$ because the surface loading of the ground with no lining $\boldsymbol{f}_{(1)}^{i}$ is zero. For the lining, displacement $\boldsymbol{u}_{(2)}$ is caused by surface loading $\boldsymbol{f}_{(2)}$ from the ground-lining interface. The displacements $\boldsymbol{u}_{(1)}^{i}$ can be determined when incident wave conditions are given. $\boldsymbol{u}_{(1)}^{r}$ and $\boldsymbol{u}_{(2)}$ can be determined by $\boldsymbol{f}_{(1)}^{r}$ and $\boldsymbol{f}_{(2)}$, respectively.

The boundary conditions at the ground-lining interface are as follows. At the discontinuous interface within the extent of the void, $\boldsymbol{f}_{(1)}=\boldsymbol{f}_{(2)}=0$ must be satisfied. At the intact interface away from the void, $\boldsymbol{f}_{(1)}+\boldsymbol{f}_{(2)}=0$ and $\left.\boldsymbol{u}_{(1)}\right|_{r=R}=\left.\boldsymbol{u}_{(2)}\right|_{r=R}$ must be satisfied (under a no-slip condition). In an analytical or ideal scenario, these boundary conditions cannot be met easily. Therefore, the point-matching method, where the boundary conditions are satisfied only at a finite set of points along the interface, is adopted. General solutions of the displacements $\left.\boldsymbol{u}_{(1)}^{r}\right|_{r=R}$ and $\left.\boldsymbol{u}_{(2)}\right|_{r=R}$ and the surface loadings $\boldsymbol{f}_{(1)}^{r}$ and $\boldsymbol{f}_{(2)}$ can be expressed as a Fourier series expansion in the circumferential and longitudinal directions. The boundary conditions can be 
approximately satisfied by determining the coefficients of the Fourier series properly.

Herein, we show the solutions under incident $\mathrm{P}$-wave or $\mathrm{S}_{2}$-wave (symmetric deformation along the $x-z$ plane) only, as derivation of the solutions under incident $\mathrm{S}_{1}$-wave (antisymmetric deformation along the $x$ - $z$ plane) is achieved in the same way.

\subsection{General solutions for the ground and lining}

\subsubsection{Solution for the primary field of the ground}

When a plane harmonic $\mathrm{P}$-wave or $\mathrm{S}_{2}$-wave impinges on a surface of a ground cavity with no lining, part of the incident wave is reflected at the cavity. The primary displacement of the ground $\boldsymbol{u}_{(1)}^{i}$, which is the superposition of an incident wave and the reflected wave, can be calculated by elastodynamics (White, 1958; Mow and Pao, 1971). At the ground-lining interface with $r=R, \boldsymbol{u}_{(1)}^{i}$ can be expressed as follows:

$$
\left.\begin{array}{c}
\left.u_{r(1)}^{i}\right|_{r=R}=\sum_{n=0}^{\infty}\left(U_{r, n(1)}^{i} \cos \gamma z+U_{r, n(1)}^{\prime i} \sin \gamma z\right) \cos n \theta e^{-i \omega t} \\
\left.u_{\theta(1)}^{i}\right|_{r=R}=\sum_{n=0}^{\infty}\left(U_{\theta, n(1)}^{i} \cos \gamma z+U_{\theta, n(1)}^{\prime i} \sin \gamma z\right) \sin n \theta e^{-i \omega t} \\
\left.u_{z(1)}^{i}\right|_{r=R}=\sum_{n=0}^{\infty}\left(U_{z, n(1)}^{i} \sin \gamma z+U_{z, n(1)}^{i} \cos \gamma z\right) \cos n \theta e^{-i \omega t}
\end{array}\right\},
$$

in which $U_{r, n(1)}^{i}, U_{\theta, n(1)}^{i}$ and $U_{z, n(1)}^{i}$ are complex constants related to symmetric deformation along the $x-y$ plane and $U_{r, n(1)}^{\prime i}, U_{\theta, n(1)}^{\prime i}$ and $U_{z, n(1)}^{\prime i}$ are those related to antisymmetric deformation along the $x-y$ plane. $\omega$ is the angular 
velocity of the incident wave and $\gamma$ is the wavenumber in the longitudinal direction. Subscript $n$ denotes the $n$th mode in the circumferential direction. When the ground property and incident wave conditions are provided, $U_{r, n(1)}^{i}$, $U_{\theta, n(1)}^{i}, U_{z, n(1)}^{i}, U_{r, n(1)}^{\prime i}, U_{\theta, n(1)}^{i}$ and $U_{z, n(1)}^{\prime i}$ are determined.

\subsubsection{Solution for the secondary field of the ground}

Displacement and traction of the ground caused by the reflected wave at the ground-lining interface with $r=R$ can also be expressed as follows $\left(e^{-i \omega t}\right.$ omitted):

$$
\left.\begin{array}{c}
\left.u_{r(1)}^{r}\right|_{r=R}=\sum_{m=0}^{\infty} \sum_{n=0}^{\infty}\left(U_{r, n m(1)}^{r} \cos \gamma_{m} z+U_{r, n m(1)}^{\prime r} \sin \gamma_{m} z\right) \cos n \theta \\
\left.\left.u_{\theta(1)}^{r}\right|_{r=R}=\sum_{m=0}^{\infty} \sum_{n=0}^{\infty}\left(U_{\theta, n m(1)}^{r} \cos \gamma_{m} z+U_{\theta, n m(1)}^{\prime r} \sin \gamma_{m} z\right) \sin n \theta\right\} \\
\left.u_{z(1)}^{r}\right|_{r=R}=\sum_{m=0}^{\infty} \sum_{n=0}^{\infty}\left(U_{z, n m(1)}^{r} \sin \gamma_{m} z+U_{z, n m(1)}^{\prime r} \cos \gamma_{m} z\right) \cos n \theta
\end{array}\right\}
$$

in which $U_{r, n m(1)}^{r}, U_{\theta, n m(1)}^{r}$, and so on are complex constants. 
Stiffness of the ground, which is defined as the relationship between displacement and traction at the ground-lining interface, can be defined for each mode. In the following, we only mention the stiffness matrix related to symmetric deformation along the $x-y$ plane, as that related to antisymmetric deformation can be derived in a similar way.

According to the theory of elastodynamics (Mow and Pao, 1971), the general solutions of displacement and traction related to symmetric deformation along the $x-y$ plane and the $x-z$ plane can be expressed as follows $\left(e^{-i \omega t}\right.$ omitted $)$ :

$$
\left.\begin{array}{r}
u_{r(1)}^{r}=\frac{1}{r} \sum_{m=0}^{\infty} \sum_{n=0}^{\infty}\left(A_{n m} \varepsilon_{11}+B_{n m} \varepsilon_{12}+C_{n m} \varepsilon_{13}\right) \cos n \theta \cos \gamma_{m} z \\
u_{\theta(1)}^{r}=\frac{1}{r} \sum_{m=0}^{\infty} \sum_{n=0}^{\infty}\left(A_{n m} \varepsilon_{21}+B_{n m} \varepsilon_{22}+C_{n m} \varepsilon_{23}\right) \sin n \theta \cos \gamma_{m} z \\
u_{z(1)}^{r}=\frac{1}{r} \sum_{m=0}^{\infty} \sum_{n=0}^{\infty}\left(A_{n m} \varepsilon_{31}+B_{n m} \varepsilon_{32}+C_{n m} \varepsilon_{33}\right) \cos n \theta \sin \gamma_{m} z
\end{array}\right\},
$$

where $\mu_{(1)}$ is the shear modulus of the ground; $A_{n m}, B_{n m}$, and $C_{n m}$ are complex constants; and $\varepsilon_{11}, \varepsilon_{12}$, and so on are given in Appendix A.

Constants $A_{n m}, B_{n m}$, and $C_{n m}$ can be removed from Eq. (5) to Eq. (9). The relationship between $U_{r, n m(1)}^{r}, U_{\theta, n m(1)}^{r}$, and $U_{z, n m(1)}^{r}$ and $F_{r, n m(1)}^{r}, F_{\theta, n m(1)}^{r}$, 
and $F_{z, n m(1)}^{r}$ can be derived as follows:

$$
\begin{aligned}
\left(\begin{array}{l}
F_{r, n m(1)}^{r} \\
F_{\theta, n m(1)}^{r} \\
F_{z, n m(1)}^{r}
\end{array}\right) & =-\frac{2 \mu}{R}\left(\begin{array}{lll}
\varepsilon_{41} & \varepsilon_{42} & \varepsilon_{43} \\
\varepsilon_{51} & \varepsilon_{52} & \varepsilon_{53} \\
\varepsilon_{61} & \varepsilon_{62} & \varepsilon_{63}
\end{array}\right)\left(\begin{array}{lll}
\varepsilon_{11} & \varepsilon_{12} & \varepsilon_{13} \\
\varepsilon_{21} & \varepsilon_{22} & \varepsilon_{23} \\
\varepsilon_{31} & \varepsilon_{32} & \varepsilon_{33}
\end{array}\right)^{-1}\left(\begin{array}{c}
U_{r, n m(1)}^{r} \\
U_{\theta, n m(1)}^{r} \\
U_{z, n m(1)}^{r}
\end{array}\right), \\
& =\left(\begin{array}{lll}
K_{r r, n m(1)} & K_{r \theta, n m(1)} & K_{r z, n m(1)} \\
K_{r \theta, n m(1)} & K_{\theta \theta, n m(1)} & K_{\theta z, n m(1)} \\
K_{r z, n m(1)} & K_{\theta z, n m(1)} & K_{z z, n m(1)}
\end{array}\right)\left(\begin{array}{l}
U_{r, n m(1)}^{r} \\
U_{\theta, n m(1)}^{r} \\
U_{z, n m(1)}^{r}
\end{array}\right)
\end{aligned}
$$

where the coefficient matrix of the right hand side in Eq. (10) is the stiffness matrix of the ground related to symmetric deformation along the $x-y$ plane.

\subsubsection{Solutions for the lining}

In this paper, for simplicity, the lining is treated as an elastic cylindrical shell, with thickness $h$ being considerably smaller than the outer radius of the lining $R$. Therefore, the mean radius of the shell is considered to be equal to $R$.

The general solutions of the lining at the ground-lining interface with $r=R$ can be expressed as follows:

$$
\left.\begin{array}{l}
u_{r(2)}=\sum_{m=0}^{\infty} \sum_{n=0}^{\infty}\left\{U_{r, n m(2)} \cos \gamma_{m} z+U_{r, n m(2)}^{\prime} \sin \gamma_{m} z\right\} \cos n \theta e^{-i \omega t} \\
\left.u_{\theta(2)}=\sum_{m=0}^{\infty} \sum_{n=0}^{\infty}\left\{U_{\theta, n m(2)} \cos \gamma_{m} z+U_{\theta, n m(2)}^{\prime} \sin \gamma_{m} z\right\} \sin n \theta e^{-i \omega t}\right\} \\
u_{z(2)}=\sum_{m=0}^{\infty} \sum_{n=0}^{\infty}\left\{U_{z, n m(2)} \sin \gamma_{m} z+U_{z, n m(2)}^{\prime} \cos \gamma_{m} z\right\} \cos n \theta e^{-i \omega t}
\end{array}\right\}
$$




$$
\begin{aligned}
f_{r(2)} & =\sum_{m=0}^{\infty} \sum_{n=0}^{\infty}\left\{F_{r, n m(2)} \cos \gamma_{m} z+F_{r, n m(2)}^{\prime} \sin \gamma_{m} z\right\} \cos n \theta e^{-i \omega t} \\
f_{\theta(2)} & \left.=\sum_{m=0}^{\infty} \sum_{n=0}^{\infty}\left\{F_{\theta, n m(2)} \cos \gamma_{m} z+F_{\theta, n m(2)}^{\prime} \sin \gamma_{m} z\right\} \sin n \theta e^{-i \omega t}\right\}, \\
f_{z(2)} & \left.=\sum_{m=0}^{\infty} \sum_{n=0}^{\infty}\left\{F_{z, n m(2)} \sin \gamma_{m} z+F_{z, n m(2)}^{\prime} \cos \gamma_{m} z\right\} \cos n \theta e^{-i \omega t}\right\}
\end{aligned}
$$

where $U_{r, n m(2)}, U_{\theta, n m(2)}$, and so on are complex constants.

The relationship between $U_{r, n m(2)}, U_{\theta, n m(2)}$, and $U_{z, n m(2)}$ and $F_{r, n m(2)}$, $F_{\theta, n m(2)}$, and $F_{z, n m(2)}$ can be derived from Eq. (11), Eq. (12), and tractiondisplacement relations for the lining (Flügge, 1973). They can be expressed as follows:

$$
\left(\begin{array}{l}
F_{r, n m(2)} \\
F_{\theta, n m(2)} \\
F_{z, n m(2)}
\end{array}\right)=\left(\begin{array}{lll}
K_{r r, n m(2)} & K_{r \theta, n m(2)} & K_{r z, n m(2)} \\
K_{r \theta, n m(2)} & K_{\theta \theta, n m(2)} & K_{\theta z, n m(2)} \\
K_{r z, n m(2)} & K_{\theta z, n m(2)} & K_{z z, n m(2)}
\end{array}\right)\left(\begin{array}{l}
U_{r, n m(2)} \\
U_{\theta, n m(2)} \\
U_{z, n m(2)}
\end{array}\right), r=R,
$$

where the coefficient matrix of the right-hand side in Eq. (13) is the stiffness matrix of the lining related to symmetric deformation along the $x-y$ plane. Coefficients of the matrix are shown in Appendix B.

\subsection{Boundary conditions}

The boundary conditions at the ground-lining interface with $r=R$ are imposed as follows. For traction-free condition:

$$
f_{r(1)}^{r}=f_{\theta(1)}^{r}=f_{z(1)}^{r}=f_{r(2)}=f_{\theta(2)}=f_{z(2)}=0 .
$$


For no-slip condition:

$$
\left.\begin{array}{ll}
\left.u_{r(1)}^{i}\right|_{r=R}+\left.u_{r(1)}^{r}\right|_{r=R}=u_{r(2)}, & f_{r(1)}^{r}+f_{r(2)}=0 \\
\left.u_{\theta(1)}^{i}\right|_{r=R}+\left.u_{\theta(1)}^{r}\right|_{r=R}=u_{\theta(2)}, & f_{\theta(1)}^{r}+f_{\theta(2)}=0 \\
\left.u_{z(1)}^{i}\right|_{r=R}+\left.u_{z(1)}^{r}\right|_{r=R}=u_{z(2)}, & f_{z(1)}^{r}+f_{z(2)}=0
\end{array}\right\} .
$$

The traction-free condition is imposed on the ground-lining interface within a void, and the no-slip condition is imposed on the ground-lining interface outside a void.

\subsection{Calculation procedure for the solutions}

Solutions can be derived on the basis of the theory described in the previous section. However, the boundary conditions cannot be easily satisfied in practice. Therefore, Fourier series are limited by a finite number of terms, and the boundary conditions are satisfied in a finite set of points (pointmatching method).

The first $N+1$ and $M+1$ terms of the infinite series (from $n=0-N$ and $m=0-M)$ are used to express the approximate solutions. $N$ points in the circumferential direction $\left(-180^{\circ} \leq \theta<180^{\circ}\right)$ and $M$ points in the longitudinal direction $(-L / 2 \leq z<L / 2)$ are selected such that they are equidistant from each other. The resulting system of inhomogeneous linear equations can be solved by matrix inversion. However, because of the large number of unknown constants, considerable time and memory are required for achieving the result. To address this drawback, the solutions are derived using an alternative strategy.

Fig. C.3 illustrates the calculation procedure. Traction and displacement vectors are represented by the vectors whose end point and starting point 
lie on the ground-lining interface, respectively. Fig. C.4 shows a flowchart of the calculation procedure to determine the solutions. The effect of a void (traction-free condition) is taken into account gradually in the loop calculation. The initial value is the solutions in case of no void. Conversion between displacement distribution and traction distribution is performed by using the stiffness matrix. The fast Fourier transform is used to reduce the calculation time when the amplitude of each mode is calculated from displacement or traction distribution. The change from the ground system D to E represents the reduction of the traction on the ground-lining interface within a void. The traction is gradually reduced, $50 \%$ at each loop iteration in this study, to avoid numerical instability. Traction in lining system A is calculated from traction in ground system E, and it is updated by the following equation: $\boldsymbol{f}_{(2)}=(1-\alpha) \boldsymbol{f}_{(2)}-\alpha \boldsymbol{f}_{(1)}$. In this study, $\alpha$ is assumed to be 0.005 .

The accuracy of the solution can be evaluated by checking whether the rigid body displacement in the ground system, $\boldsymbol{u}_{(1)}^{\text {rigid }}$, is finally zero. Under Pwave or $\mathrm{S}_{2}$-wave incidence (symmetric deformation along the $x$ - $z$ plane), the rigid body displacement in the $x$-direction is evaluated by $U_{r, 10(1)}+U_{\theta, 10(1)}$. In this paper, the calculation is stopped when the normalized displacement $\left|\boldsymbol{u}_{(1)}^{\text {rigid }}\right| /\left|\boldsymbol{u}_{(1)}^{\text {inc }}\right|$ becomes less than $10^{-20}$.

\subsection{Stress of the lining}

Axial thrust $n_{\theta \theta}, n_{\theta z}$, and $n_{z z}$ and bending moment $m_{\theta \theta}, m_{\theta z}$, and $m_{z z}$ in the lining can be calculated from the displacement of the lining, as expressed 
(Flügge, 1973) below:

$$
\left.\begin{array}{l}
n_{\theta \theta}=\sum_{m=0}^{\infty} \sum_{n=0}^{\infty} N_{\theta \theta, n m} \cos n \theta \cos \gamma_{m} z e^{-i \omega t} \\
n_{z z}=\sum_{m=0}^{\infty} \sum_{n=0}^{\infty} N_{z z, n m} \cos n \theta \cos \gamma_{m} z e^{-i \omega t} \\
n_{\theta z}=\sum_{m=0}^{\infty} \sum_{n=0}^{\infty} N_{\theta z, n m} \sin n \theta \sin \gamma_{m} z e^{-i \omega t}
\end{array}\right\},
$$

where $N_{\theta \theta, n m}, M_{\theta \theta, n m}$, and so on are shown in Appendix C. The sign convention for thrust and moment in the lining is shown in Fig. C.5.

Stress at the surface of the lining can be approximated as follows:

$$
\left.\begin{array}{rl}
\sigma_{\theta \theta(2)} & \approx \frac{1}{h} n_{\theta \theta} \pm \frac{h}{2 I} m_{\theta \theta} \\
\sigma_{z z(2)} & \left.\approx \frac{1}{h-\frac{I}{R^{2}}}\left\{\left(1 \pm \frac{h}{2 R}\right) n_{z z}+\left( \pm \frac{h^{2}}{2 I}+\frac{1}{R}\right) m_{z z}\right\}\right\}, \\
\sigma_{\theta z(2)} & \approx \frac{1}{h} n_{\theta z} \pm \frac{h}{2 I} m_{\theta z} \\
I & =\frac{h^{3}}{12}
\end{array}\right\}
$$

where $I$ is the moment of inertia of the lining. As for \pm in Eq. (18), the positive and negative signs are chosen for the inner and outer surfaces of the lining, respectively. 


\section{Results and discussion}

Table. C.1 lists the material properties required for the numerical calculations. We assume that the Shinkansen tunnel is in soft ground because tunnels are severely damaged by earthquakes are usually constructed in soft ground. The ratio of Young's modulus of the ground and the lining is 0.01:1. The pressure wave velocity and shear wave velocity in the ground are approximately $450 \mathrm{~m} / \mathrm{s}$ and $240 \mathrm{~m} / \mathrm{s}$, respectively. Incident wave frequency $f$ is assumed to be $1.0 \mathrm{~Hz}$ as referenced from the velocity response spectrum observed on the base rock during the 1995 Kobe earthquake.

In the following, to satisfy the boundary conditions, $N=128$ points in $0^{\circ} \leq \theta<360^{\circ}$ are selected in the circumferential direction keeping approximately $0.25 \mathrm{~m}$ distance between two points. In the longitudinal direction, $M$ points are selected such that $M$ is the minimum number in power of two with the distance between two points being less than $0.25 \mathrm{~m}$. For example, $M$ is 4096 under S-wave incidence with $f=1.0 \mathrm{~Hz}$ and $\phi=75^{\circ}$.

\subsection{Solutions with no void behind the lining}

To understand the basic seismic response of a tunnel, the case of no void behind the lining is investigated before the case with a void. When there is no void, the boundary condition in Eq. (15) can be satisfied strictly. Solutions are calculated using the first eleven terms for the circumferential direction $(n=0-10)$ and only one term for the longitudinal direction $(m=1)$.

\subsubsection{Characteristic deformation modes}

The wavelength of the incident wave is sufficiently longer than the tunnel radius; therefore, the lining deformation is predominant in the three lowest 
modes that correspond to the circumferential mode, i.e., $n=0,1$, and 2 .

Fig. C.6 shows the characteristic deformation modes of the lining under $\mathrm{S}_{2}$-wave incidence at an incident angle $\phi$ of $75^{\circ}$. The amplitude of the incident shear wave displacement $\mathbf{u}_{\mathrm{s}_{2}(1)}^{\mathrm{inc}}$ is $1.0 \mathrm{~m}$ in the ground. The calculated displacement is multiplied by the number given in the upper right corner of the legend. Note that $x$-direction displacement is emphasized in these figures because the ranges of the $x$-axis and $z$-axis are $\pm 10 \mathrm{~m}$ and $\pm 1000 \mathrm{~m}$, respectively. The zeroth, first, and second modes represent compression-extension deformation, longitudinal bending deformation on the $x-z$ plane, and the ovaling deformation of the tunnel cross-section, respectively. In this case, the displacements in the $z$-direction for the zeroth mode, the $x$-direction for the first mode, and the $x$-direction for the second mode are approximately $0.85,0.26$, and $0.03 \mathrm{~m}$, respectively. Therefore, the $z$-direction displacement for the zeroth mode is predominant in this case.

Fig. C.7 shows characteristic deformation modes of the lining under the $\mathrm{S}_{1}$-wave incidence at an incident angle $\phi$ of $75^{\circ}$. The amplitude of the incident shear wave displacement $\mathbf{u}_{\mathrm{s}_{1}(1)}^{\text {inc }}$ is $1.0 \mathrm{~m}$. Each figure shows the displacement of the lining at maximum displacement of each mode. The zeroth, first, and second modes represent the torsional deformation, longitudinal bending deformation and ovaling deformations, respectively. Except for the zeroth mode, all deformation modes under $\mathrm{S}_{1}$-wave and $\mathrm{S}_{2}$-wave incidence are similar with the difference being the deformation direction. 
3.1.2. Relationships between incident angle and stress distribution of the lining

When the seismic wave is incident on the tunnel, the lining stress in the inner surface maximizes. Therefore, we investigate the stress of the lining inner surface to evaluate the seismic response of the tunnel. The stress is normalized by the incident $\mathrm{P}$-wave- or S-wave-induced maximum principal stress of the ground without excavation (infinite ground).

Fig. C.8 shows the maximum stress distribution of the inner surface of the lining under $\mathrm{P}$-wave incidence. At an incident angle $\phi$ of $15^{\circ}, \sigma_{z z}^{*}$ is predominant and is almost uniformly distributed in the lining. At $\phi=75^{\circ}, \sigma_{\theta \theta}^{*}$ is predominant and is maximum at $\theta= \pm 90^{\circ}$. Fig. C.9 shows the maximum stress of the inner surface of the lining at $\theta=90^{\circ}$ and the contribution of each mode. In the legend, $n$ denotes the terms of the modes used for the calculation. When $\phi$ is small, $\sigma_{z z}^{*}$ by the zeroth mode is predominant, whereas when $\phi$ is large, $\sigma_{\theta \theta}^{*}$ by the superposition of the zeroth mode and the second mode is predominant. The maximum lining stress under P-wave incidence occurs when $\phi$ is small.

Fig. C.10 shows the maximum stress distribution of the inner surface of the lining under $\mathrm{S}_{1}$-wave incidence. When $\phi$ is $15^{\circ}, \sigma_{z z}^{*}$ and $\sigma_{\theta z}^{*}$ are predominant and become maximum at $\theta= \pm 90^{\circ}$ and $0^{\circ}$ and $\pm 180^{\circ}$, respectively. When $\phi$ is $75^{\circ}, \sigma_{\theta \theta}^{*}$ is predominant and becomes maximum at $\theta= \pm 45^{\circ}$ and $\pm 135^{\circ}$. Fig. C.11 shows the maximum stress of the inner surface of the lining at $\theta=45^{\circ}$ and the contribution of each mode. When $\phi$ is small, $\sigma_{z z}^{*}$ by the first mode is predominant, whereas when $\phi$ is large, $\sigma_{\theta \theta}^{*}$ by the second mode is predominant. $\sigma_{\theta \theta}^{*}$ for a large incident angle is larger than $\sigma_{z z}^{*}$ for a 
small incident angle. The maximum lining stress occurs $\phi$ is $90^{\circ} . \sigma_{\theta \theta}^{*}$ can be approximately related to $\sin \phi$.

Fig. C.12 shows the maximum stress distribution of the inner surface of the lining under $\mathrm{S}_{2}$-wave incidence. $\sigma_{z z}^{*}$ is predominant for both incident angles. When $\phi$ is $15^{\circ}, \sigma_{z z}^{*}$ becomes maximum at $\theta=0^{\circ}$ and $\pm 180^{\circ}$. When $\phi$ is $75^{\circ}, \sigma_{z z}^{*}$ is almost uniformly distributed. Fig. C.13 shows the maximum stress of the inner surface of the lining at $\theta=0^{\circ}$ and the contribution of each mode. When $\phi$ is small, $\sigma_{z z}^{*}$ by the first mode is predominant, whereas when $\phi$ is large, $\sigma_{z z}^{*}$ by the zeroth mode is predominant. Maximum lining stress occurs when $\phi$ is large. When the tunnel is sufficiently flexible relative to the surrounding ground and completely follows the ground deformation (freefield deformation), $\sigma_{z z}^{*}$ by the zeroth mode becomes maximum at $\phi=45^{\circ}$ (Yeh, 1974; Hashash et al., 2001). When the tunnel is not sufficiently flexible, $\sigma_{z z}^{*}$ becomes maximum at $45^{\circ} \leq \phi<90^{\circ}$ by the ground-lining interaction. This is because the stiffness of the lining decreases more rapidly than that of the ground as the wavelength along the tunnel axis increases. The first mode does not tend to cause large stress concentration in the lining because of the long wavelength of the incident shear wave and the relatively small curvature of the lining caused by the deformation.

\subsubsection{Comparison between maximum circumferential strain and longitudinal strain}

The discussion in 3.1.2 considers only the specific conditions given in Table. C.1. This section discusses the solutions for various Young's ratios of the ground and frequency. In general, a seismic wave is incident on the tunnel at a large angle, and the amplitude of the S-wave is larger than that 
of the P-wave. Therefore, solutions for S-wave incidence at large incident angles are considered.

Fig. C.14 shows the maximum $\varepsilon_{\theta \theta}^{*}$ and maximum $\varepsilon_{z z}^{*}$ for various Young's ratios of the ground and frequency. The solutions are evaluated in terms of strain, rather than stress, in order to better evaluate the effect of varying Young's modulus of the ground over a wide range. The strain is normalized by the S-wave-induced maximum shear strain of the ground without excavation. $\varepsilon_{\theta \theta}^{*}$ is the maximum circumferential strain on the inner surface of the lining when the $\mathrm{S}_{1}$-wave is incident on the tunnel at $\phi=90^{\circ} . \varepsilon_{z z}^{*}$ is the maximum longitudinal strain on the inner surface of the lining when the $\mathrm{S}_{2}$-wave is incident on the tunnel at $45^{\circ} \leq \phi<90^{\circ}$.

All three curves of $\varepsilon_{\theta \theta}^{*}$ tend downward to the left of the figure. $\varepsilon_{\theta \theta}^{*}$ is influenced by the relative stiffness of the ground and the lining, but not by the frequency of the shear wave. This is because the shear wavelength is much larger than the tunnel radius. For example, the shear wavelength is about 18 times larger than the tunnel radius at a frequency of $5.0 \mathrm{~Hz}$ and Young's ratio of the ground of 1.0 GPa.

In contrast, $\varepsilon_{z z}^{*}$ is influenced by both relative stiffness and frequency. $\varepsilon_{z z}^{*}$ in the weak ground is strongly affected by the frequency. It decreases as Young's modulus of the ground decreases and frequency increases because, as mentioned earlier, the stiffness of the lining decreases more rapidly than that of the ground with increasing wavelength along the tunnel axis. When the tunnel is sufficiently flexible relative to the surrounding ground and completely follows ground deformation, $\varepsilon_{z z}^{*}$ is 0.5 .

The maximum $\varepsilon_{z z}^{*}$ tends to be larger than $\varepsilon_{\theta \theta}^{*}$ when Young's ratio of the 
ground is less than 3.0 GPa (approximately one-tenth of that of the lining). Therefore, seismic damage of the tunnel in the weak ground is probablyi caused by both the $\mathrm{S}_{1}$-wave and $\mathrm{S}_{2}$-wave.

\subsection{Solutions with a void behind the lining}

Figs. C.9, C.11 and C.13 show that the stress of the lining is mainly caused by the first three modes (zeroth, first, and second). Especially, the second mode is predominant for circumferential stress, while the zeroth mode is predominant for longitudinal stress. In the following, the effect of a void for these modes is considered. We consider a relatively large void, ranging $60^{\circ}\left(-30^{\circ} \leq \theta \leq 30^{\circ}\right)$ in the circumferential direction and $3 R(-7.5 \mathrm{~m} \leq z<$ $7.5 \mathrm{~m})$ in the longitudinal direction. The stress is normalized by the incident wave-induced maximum principal stress of the ground without excavation.

Fig. C.15 shows the circumferential stress distributions of the inner, middle, and outer surfaces of the lining at a cross-section of $z=0$ when the maximum principal stress at the cross-section is maximum under $\mathrm{S}_{1}$-wave incidence at an incident angle $\phi$ of $75^{\circ}$. The frequency $f$ is $1.0 \mathrm{~Hz}$ and 5.0 Hz. The effect of a void is the largest at a cross-section $z=0$, which is the center of the void in the longitudinal direction. Only axial thrust occurs on the middle surface of the lining, and the stress difference between the middle surface and the inner or outer surface of the lining is caused by the bending moment. The circumferential stress is mainly caused by the second mode deformation when there is no void, and the circumferential stress caused by the bending moment is higher than by the axial thrust. A void causes little additional circumferential stress concentration on the lining because the presence of a void causes a negative effect on the lining owing to the change 
in the stress state of the lining from axial thrust to bending moment (Yasuda et al., 2017). The frequency, $f=1.0 \mathrm{~Hz}$ or $5.0 \mathrm{~Hz}$, barely has any effect on the circumferential stress distribution, regardless of the presence of a void, because wavelength of the S-wave is considerably longer than the void size.

Fig. C.16 shows the circumferential stress distributions of the inner, middle, and outer surfaces of the lining at the cross-section $z=0$ when the principal stress at the cross-section is maximum under $\mathrm{P}$-wave incidence. The circumferential stress is caused by the zeroth mode and the second mode deformation when there is no void. The void causes additional stress concentration on the lining. However, stress concentration around the void decreases. The presence of a void does not affect the maximum principal stress as this stress occurs outside the void.

Fig. C.17 shows the longitudinal stress distributions of the inner, middle, and outer surfaces of the lining at the cross-section $z=0$ under $\mathrm{S}_{2}$-wave incidence. The longitudinal stress is mainly caused by the zeroth mode deformation when there is no void. The longitudinal stress caused by the axial thrust is higher than that caused by the bending moment. However, unlike the circumferential stress, the void causes little additional stress concentration on the lining because large longitudinal stress is caused by the accumulation of $f_{z(2)}\left(=\sigma_{r z(2)}\right)$, and the range of applied $f_{z(2)}$ is considerably larger than the void size. For example, in this case, the wavelength of the applied $f_{z(2)}$ is approximately $900 \mathrm{~m}$.

Past experiences in Japan suggest a high correlation between the presence of voids and seismic damage of tunnels. However, as shown in Figs. C.15, C.16, and C.17, the presence of the void does not lead to a large stress 
concentration on the lining. Incidentally, a void does not cause high circumferential stress concentration on the lining under shear loading, but does under isotropic compression such as loading in construction and elevating earth pressure after construction (Yasuda et al., 2017); and old tunnels that were constructed using conventional method (tunnelling method before New Austrian tunnelling method) often have large stress concentration in the lining before an earthquake. Taking this fact into consideration, large stress concentration caused by the voids before an earthquake is considered to be highly correlated with the seismic damage of tunnels. Therefore, to prevent the seismic damage of tunnels, the lining of the parts where large stress concentration has already occurred should be reinforced.

\section{Conclusions and remarks}

This study presented three-dimensional elastic solutions for a deep cylindrical tunnel with a void behind the lining and subjected to obliquely incident seismic waves. The three-dimensional seismic response of the tunnel and the effects of a void on the response were investigated. The following conclusions can be drawn from this study:

(1) Because the incident wavelength is sufficiently longer than the tunnel radius, the lowest three, namely, zeroth, first, and second, modes are predominant on the lining deformation. These modes represent the compression-extension deformation or torsional deformation, the longitudinal bending deformation, and the ovaling deformation modes, respectively. 
(2) The circumferential stress and strain of the lining are influenced by the relative stiffness of the ground and the lining and are not influenced by the wavelength of the incident wave because the wavelength is considarably larger than the tunnel radius. In contrast, the longitudinal stress and strain of the lining are influenced by both the relative stiffness and the wavelength along the tunnel axis. Therefore, seismic damage of the tunnel in a weak ground is probably caused by shear waves with their displacement components not only parallel to the tunnel crosssection but also located on the plane that includes tunnel axis.

(3) A void causes little additional circumferential stress concentration because the presence of a void negatively affects the lining owing to the change in the stress state of the lining from axial thrust to bending moment.

(4) A void causes little additional longitudinal stress concentration because a longitudinal stress is caused by the accumulation of longitudinal shear stress and the applied stress range is considerably large compared to the void size.

(5) Large stress concentration caused by voids before an earthquake is considered to be highly correlated with seismic damage of tunnels. Therefore, the lining where large stress concentration has already occurred should be reinforced to prevent seismic damage of the tunnel. 


\section{Appendix A. General solutions of the ground system}

In the absence of a body force, the displacement equation of the motion is

$$
\left(\lambda_{(1)}+\mu_{(1)}\right) \nabla \nabla \cdot \boldsymbol{u}+\mu_{(1)} \nabla^{2} \boldsymbol{u}=\rho_{(1)} \ddot{\boldsymbol{u}},
$$

where $\lambda_{(1)}$ and $\mu_{(1)}$ are Lamé constants of the ground and $\rho_{(1)}$ is the density of the ground.

Displacement solutions of Eq. (A.1) are given by (Mow and Pao, 1971)

$$
\left.\begin{array}{l}
u_{r(1)}=\frac{\partial \varphi}{\partial r}+\frac{1}{r} \frac{\partial \psi}{\partial \theta}+\frac{1}{k_{s(1)}} \frac{\partial^{2} \chi}{\partial r \partial z} \\
u_{\theta(1)}=\frac{1}{r} \frac{\partial \varphi}{\partial \theta}-\frac{\partial \psi}{\partial r}+\frac{1}{k_{s(1)} r} \frac{\partial^{2} \chi}{\partial \theta \partial z} \\
u_{z(1)}=\frac{\partial \varphi}{\partial z}-\frac{1}{k_{s(1)}}\left\{\frac{1}{r} \frac{\partial}{\partial r}\left(r \frac{\partial \chi}{\partial r}\right)+\frac{1}{r^{2}} \frac{\partial^{2} \chi}{\partial \theta^{2}}\right\}
\end{array}\right\},
$$

where $k_{s(1)}$ is the shear wavenumber in the ground; $\varphi$ is the pressure wave potential; and $\psi$ and $\chi$ are the shear wave potentials. These satisfy the wave equation as follows:

$$
\left.\begin{array}{r}
c_{p(1)}^{2} \nabla^{2} \varphi=\ddot{\varphi} \\
c_{s(1)}^{2} \nabla^{2} \psi=\ddot{\psi} \\
c_{s(1)}^{2} \nabla^{2} \chi=\ddot{\chi}
\end{array}\right\},
$$

where $c_{p(1)}$ and $c_{s(1)}$ are the pressure wave velocity and the shear wave velocity in the ground, respectively. 
Solutions of the scalar potentials $\varphi, \psi$, and $\chi$ can be expressed as follows:

$$
\left.\begin{array}{c}
\left.\varphi=A_{n m} H_{n}^{(1)}\left(\alpha_{m(1)} r\right)\left\{\begin{array}{l}
\cos n \theta \\
\sin n \theta
\end{array}\right\}\left[\begin{array}{l}
\cos \gamma_{m} \\
\sin \gamma_{m}
\end{array}\right] e^{-i \omega t}\right\} \\
\left.\psi=B_{n m} H_{n}^{(1)}\left(\beta_{m(1)} r\right)\left\{\begin{array}{l}
\sin n \theta \\
\cos n \theta
\end{array}\right\}\left[\begin{array}{l}
\cos \gamma_{m} \\
\sin \gamma_{m}
\end{array}\right] e^{-i \omega t}\right\}, \\
\left.\chi=C_{n m} H_{n}^{(1)}\left(\beta_{m(1)} r\right)\left\{\begin{array}{l}
\cos n \theta \\
\sin n \theta
\end{array}\right\}\left[\begin{array}{l}
\sin \gamma_{m} \\
\cos \gamma_{m}
\end{array}\right] e^{-i \omega t}\right\} \\
\alpha_{m(1)}^{2}=k_{p(1)}^{2}-\gamma_{m}^{2} \\
\beta_{m(1)}^{2}=k_{s(1)}^{2}-\gamma_{m}^{2}
\end{array}\right\},
$$

where $A_{n m}, B_{n m}$, and $C_{n m}$ are complex constants. $H_{n}^{(1)}$ denotes the Hankel functions of the first order $n$, and $k_{p(1)}$ is the pressure wavenumber in the ground.

From the Eqs. (A.2) - (A.6), the ground displacement can be expressed as follows:

$$
\left.\begin{array}{l}
u_{r(1)}=\frac{A_{n m} \varepsilon_{11}+B_{n m} \varepsilon_{12}+C_{n m} \varepsilon_{13}}{r}\left\{\begin{array}{c}
\cos n \theta \\
\sin n \theta
\end{array}\right\}\left[\begin{array}{l}
\cos \gamma_{m} \\
\sin \gamma_{m}
\end{array}\right] e^{-i \omega t} \\
\left.u_{\theta(1)}=\frac{A_{n m} \varepsilon_{21}+B_{n m} \varepsilon_{22}+C_{n m} \varepsilon_{23}}{r}\left\{\begin{array}{c}
\sin n \theta \\
\cos n \theta
\end{array}\right\}\left[\begin{array}{l}
\cos \gamma_{m} \\
\sin \gamma_{m}
\end{array}\right] e^{-i \omega t}\right\}, \\
u_{z(1)}=\frac{A_{n m} \varepsilon_{31}+B_{n m} \varepsilon_{32}+C_{n m} \varepsilon_{33}}{r}\left\{\begin{array}{c}
\cos n \theta \\
\sin n \theta
\end{array}\right\}\left[\begin{array}{c}
\sin \gamma_{m} \\
\cos \gamma_{m}
\end{array}\right] e^{-i \omega t}
\end{array}\right\}
$$




$$
\left.\begin{array}{l}
\varepsilon_{11}=\alpha_{m(1)} r H_{n-1}^{(1)}\left(\alpha_{m(1)} r\right)-n H_{n}^{(1)}\left(\alpha_{m(1)} r\right) \\
\varepsilon_{12}=\{ \pm n\} H_{n}^{(1)}\left(\beta_{m(1)} r\right) \\
\varepsilon_{13}=\frac{\left[ \pm \gamma_{m}\right]}{k_{s(1)}}\left\{\beta_{m(1)} r H_{n-1}^{(1)}\left(\beta_{m(1)} r\right)-n H_{n}^{(1)}\left(\beta_{m(1)} r\right)\right\} \\
\varepsilon_{21}=\{\mp n\} H_{n}^{(1)}\left(\alpha_{m(1)} r\right) \\
\varepsilon_{22}=-\left\{\beta_{m(1)} r H_{n-1}^{(1)}\left(\beta_{m(1)} r\right)-n H_{n}^{(1)}\left(\beta_{m(1)} r\right)\right\} \\
\varepsilon_{23}=\frac{\{\mp n\}\left[ \pm \gamma_{m}\right]}{k_{s(1)}} H_{n}^{(1)}\left(\beta_{m(1)} r\right) \\
\varepsilon_{31}=\left[\mp \gamma_{m}\right] r H_{n}^{(1)}\left(\alpha_{m(1)} r\right) \\
\varepsilon_{32}=0 \\
\varepsilon_{33}=\frac{\beta_{m(1)}^{2} r}{k_{s(1)}} H_{n}^{(1)}\left(\beta_{m(1)} r\right)
\end{array}\right\} .
$$

Using the displacement given by Eq. (A.7), the displacement-strain relationship, and the strain-stress relationship, the ground stress can be expressed as follows:

$$
\left.\begin{array}{c}
\sigma_{r r(1)}=\frac{2 \mu_{(1)}\left(A_{n m} \varepsilon_{41}+B_{n m} \varepsilon_{42}+C_{n m} \varepsilon_{43}\right)}{r^{2}}\left\{\begin{array}{l}
\cos n \theta \\
\sin n \theta
\end{array}\right\}\left[\begin{array}{l}
\cos \gamma_{m} \\
\sin \gamma_{m}
\end{array}\right] e^{-i \omega t} \\
\left.\sigma_{r \theta(1)}=\frac{2 \mu_{(1)}\left(A_{n m} \varepsilon_{51}+B_{n m} \varepsilon_{52}+C_{n m} \varepsilon_{53}\right)}{r^{2}}\left\{\begin{array}{l}
\sin n \theta \\
\cos n \theta
\end{array}\right\}\left[\begin{array}{l}
\cos \gamma_{m} \\
\sin \gamma_{m}
\end{array}\right] e^{-i \omega t}\right\}, \\
\sigma_{r z(1)}=\frac{2 \mu_{(1)}\left(A_{n m} \varepsilon_{61}+B_{n m} \varepsilon_{62}+C_{n m} \varepsilon_{63}\right)}{r^{2}}\left\{\begin{array}{l}
\cos n \theta \\
\sin n \theta
\end{array}\right\}\left[\begin{array}{l}
\sin \gamma_{m} \\
\cos \gamma_{m}
\end{array}\right] e^{-i \omega t}
\end{array}\right\}
$$




$$
\left.\begin{array}{rl}
\varepsilon_{41}= & -\alpha_{m(1)} r H_{n-1}^{(1)}\left(\alpha_{m(1)} r\right)+\left(n^{2}+n-\frac{k_{s(1)}^{2} r^{2}}{2}+\gamma_{m}^{2} r^{2}\right) H_{n}^{(1)}\left(\alpha_{m(1)} r\right) \\
\varepsilon_{42}= & \{ \pm n\}\left\{\beta_{m(1)} r H_{n-1}^{(1)}\left(\beta_{m(1)} r\right)-(n+1) H_{n}^{(1)}\left(\beta_{m(1)} r\right)\right\} \\
\varepsilon_{43}= & \frac{\left[ \pm \gamma_{m}\right]}{k_{s(1)}}\left\{-\beta_{m(1)} r H_{n-1}^{(1)}\left(\beta_{m(1)} r\right)+\left(n^{2}+n-\beta_{m(1)}^{2} r^{2}\right) H_{n}^{(1)}\left(\beta_{m(1)} r\right)\right\} \\
\varepsilon_{51}=\{\mp n\}\left\{\alpha_{m(1)} r H_{n-1}^{(1)}\left(\alpha_{m(1)} r\right)-(n+1) H_{n}^{(1)}\left(\alpha_{m(1)} r\right)\right\} \\
\varepsilon_{52}=\beta_{m(1)} r H_{n-1}^{(1)}\left(\beta_{m(1)} r\right)-\left(n^{2}+n-\frac{\beta_{m(1)}^{2} r^{2}}{2}\right) H_{n}^{(1)}\left(\beta_{m(1)} r\right) \\
\varepsilon_{53}=\frac{\{\mp n\}\left[ \pm \gamma_{m}\right]}{k_{s(1)}\left\{\beta_{m(1)} r H_{n-1}^{(1)}\left(\beta_{m(1)} r\right)-(n+1) H_{n}^{(1)}\left(\beta_{m(1)} r\right)\right\}} \\
\varepsilon_{61}=\left[\mp \gamma_{m}\right] r\left\{\alpha_{m(1)} r H_{n-1}^{(1)}\left(\alpha_{m(1)} r\right)-n H_{n}^{(1)}\left(\alpha_{m(1)} r\right)\right\} \\
\varepsilon_{62}=\frac{\{ \pm n\}\left[\mp \gamma_{m}\right] r H_{n}^{(1)}\left(\beta_{m(1)} r\right)}{2} \\
\varepsilon_{63}=\frac{\beta_{m(1)}^{2} r^{2}-\gamma_{m}^{2} r^{2}}{2 k_{s(1)} r}\left\{\beta_{m(1)} r H_{n-1}^{(1)}\left(\beta_{m(1)} r\right)-n H_{n}^{(1)}\left(\beta_{m(1)} r\right)\right\}
\end{array}\right\}
$$




\section{Appendix B. General solutions of the lining system}

Displacement and traction in the cylindrical shell can be expressed as follows:

$$
\begin{aligned}
& \left.u_{r(2)}=U_{r, n m(2)}\left\{\begin{array}{c}
\cos n \theta \\
\sin n \theta
\end{array}\right\}\left[\begin{array}{c}
\cos \gamma_{m} \\
\sin \gamma_{m}
\end{array}\right] e^{-i \omega t}\right) \\
& \left.u_{\theta(2)}=U_{\theta, n m(2)}\left\{\begin{array}{c}
\sin n \theta \\
\cos n \theta
\end{array}\right\}\left[\begin{array}{c}
\cos \gamma_{m} \\
\sin \gamma_{m}
\end{array}\right] e^{-i \omega t}\right\} \text {, } \\
& \left.u_{z(2)}=U_{z, n m(2)}\left\{\begin{array}{c}
\cos n \theta \\
\sin n \theta
\end{array}\right\}\left[\begin{array}{c}
\sin \gamma_{m} \\
\cos \gamma_{m}
\end{array}\right] e^{-i \omega t}\right\} \\
& \left.f_{r(2)}=\sigma_{r r(2)}=F_{r, n m(2)}\left\{\begin{array}{l}
\cos n \theta \\
\sin n \theta
\end{array}\right\}\left[\begin{array}{l}
\cos \gamma_{m} \\
\sin \gamma_{m}
\end{array}\right] e^{-i \omega t}\right) \\
& \left.f_{\theta(2)}=\sigma_{r \theta(2)}=F_{\theta, n m(2)}\left\{\begin{array}{l}
\sin n \theta \\
\cos n \theta
\end{array}\right\}\left[\begin{array}{l}
\cos \gamma_{m} \\
\sin \gamma_{m}
\end{array}\right] e^{-i \omega t}\right\} \text {, } \\
& \left.f_{z(2)}=\sigma_{r z(2)}=F_{z, n m(2)}\left\{\begin{array}{c}
\cos n \theta \\
\sin n \theta
\end{array}\right\}\left[\begin{array}{c}
\sin \gamma_{m} \\
\cos \gamma_{m}
\end{array}\right] e^{-i \omega t}\right\}
\end{aligned}
$$

where $U_{r, n m(2)}, U_{\theta, n m(2)}$, and so on are constants.

The relationship between $U_{r, n m(2)}, U_{\theta, n m(2)}$, and $U_{z, n m(2)}$ and $F_{r, n m(2)}$, $F_{\theta, m(2)}$, and $F_{z, n m(2)}$ can be derived from Eq. (B.1), Eq. (B.2), and the traction-displacement relation in the shell (Flügge, 1973). It can be expressed as follows:

$$
\left(\begin{array}{l}
F_{r, n m(2)} \\
F_{\theta, n m(2)} \\
F_{z, n m(2)}
\end{array}\right)=\left(\begin{array}{lll}
K_{r r, n m(2)} & K_{r \theta, n m(2)} & K_{r z, n m(2)} \\
K_{r \theta, n m(2)} & K_{\theta \theta, n m(2)} & K_{\theta z, n m(2)} \\
K_{r z, n m(2)} & K_{\theta z, n m(2)} & K_{z z, n m(2)}
\end{array}\right)\left(\begin{array}{c}
U_{r, n m(2)} \\
U_{\theta, n m(2)} \\
U_{z, n m(2)}
\end{array}\right)
$$




$$
\begin{aligned}
& K_{r r, n m(2)}= \frac{D}{R^{2}}+\frac{K}{R^{4}}\left\{\left(\gamma_{m} R\right)^{4}+2 n^{2}\left(\gamma_{m} R\right)^{2}+\left(n^{2}-1\right)^{2}\right\}+\rho_{(2)} h \omega^{2} \\
& K_{r \theta, n m(2)}=\{ \pm n\}\left[\frac{D}{R^{2}}+\frac{K}{R^{4}}\left\{\frac{3-\nu_{(2)}}{2}\left(\gamma_{m} R\right)^{2}\right\}\right] \\
& K_{r z, n m(2)}=\left[ \pm \gamma_{m}\right] R\left[\frac{D}{R^{2}} \nu_{(2)}+\frac{K}{R^{4}}\left\{-\frac{1-\nu_{(2)}}{2} n^{2}+\left(\gamma_{m} R\right)^{2}\right\}\right] \\
& K_{\theta \theta, n m(2)}=\frac{D}{R^{2}}\left\{n^{2}+\frac{1-\nu_{(2)}}{2}\left(\gamma_{m} R\right)^{2}\right\} \\
&+\frac{K}{R^{4}}\left\{\frac{3\left(1-\nu_{(2)}\right)}{2}\left(\gamma_{m} R\right)^{2}\right\}+\rho_{(2)} h \omega^{2} \\
& K_{\theta z, n m(2)}=\left[ \pm \gamma_{m}\right] R\left(\frac{D}{R^{2}} \frac{1+\nu_{(2)}}{2}\{ \pm n\}\right) \\
&\left.K_{z z, n m(2)}=\frac{D}{R^{2}}\left\{\frac{1-\nu_{(2)}}{2} n^{2}+\left(\gamma_{m} R\right)^{2}\right\}+\frac{K}{R^{4}}\left(\frac{1-\nu_{(2)}}{2} n^{2}\right)+\rho_{(2)} h \omega^{2}\right\}
\end{aligned}
$$

$$
\left.\begin{array}{l}
D=\frac{E_{(2)} h}{1-\nu_{(2)}^{2}} \\
K=\frac{E_{(2)} h^{3}}{12\left(1-\nu_{(2)}^{2}\right)}
\end{array}\right\},
$$

where the coefficient matrix of the right hand side in Eq. (B.3) is the stiffness matrix of the lining. $E_{(2)}, \nu_{(2)}$, and $\rho(2)$ are the Young's modulus, Poisson's ratio, and density of the lining, respectively. $D$ is the extensional rigidity and $K$ is the flexural rigidity.

\section{Appendix C. Axial thrust and bending moment in the cylindrical shell}

When the displacement and traction in the cylindrical shell are expressed as Eqs. (B.1) and (B.2), axial thrust $n_{\theta \theta}, n_{\theta z}$ and $n_{z z}$, and bending moment $m_{\theta \theta}, m_{\theta z}$ and $m_{z z}$ can be calculated as follows: 


$$
\begin{aligned}
& \left.n_{\theta \theta}=N_{\theta \theta, n m}\left\{\begin{array}{c}
\cos n \theta \\
\sin n \theta
\end{array}\right\}\left[\begin{array}{c}
\cos \gamma_{m} \\
\sin \gamma_{m}
\end{array}\right] e^{-i \omega t}\right) \\
& \left.n_{z z}=N_{z z, n m}\left\{\begin{array}{l}
\cos n \theta \\
\sin n \theta
\end{array}\right\}\left[\begin{array}{l}
\cos \gamma_{m} \\
\sin \gamma_{m}
\end{array}\right] e^{-i \omega t}\right\} \text {, } \\
& \left.n_{\theta z}=N_{\theta z, n m}\left\{\begin{array}{c}
\sin n \theta \\
\cos n \theta
\end{array}\right\}\left[\begin{array}{c}
\sin \gamma_{m} \\
\cos \gamma_{m}
\end{array}\right] e^{-i \omega t}\right\} \\
& \left.m_{\theta \theta}=M_{\theta \theta, n m}\left\{\begin{array}{l}
\cos n \theta \\
\sin n \theta
\end{array}\right\}\left[\begin{array}{l}
\cos \gamma_{m} \\
\sin \gamma_{m}
\end{array}\right] e^{-i \omega t}\right) \\
& \left.m_{z z}=M_{z z, n m}\left\{\begin{array}{l}
\cos n \theta \\
\sin n \theta
\end{array}\right\}\left[\begin{array}{l}
\cos \gamma_{m} \\
\sin \gamma_{m}
\end{array}\right] e^{-i \omega t}\right\} \text {, } \\
& \left.m_{\theta z}=M_{\theta z, n m}\left\{\begin{array}{c}
\sin n \theta \\
\cos n \theta
\end{array}\right\}\left[\begin{array}{l}
\sin \gamma_{m} \\
\cos \gamma_{m}
\end{array}\right] e^{-i \omega t}\right\}
\end{aligned}
$$

$$
\left.\begin{array}{rl}
N_{\theta \theta, n m} & =\frac{D}{R}\left(U_{r, n m}\{ \pm n\} U_{\theta, n m}\left[ \pm \gamma_{m}\right] \nu_{(2)} R U_{z, n m}\right)+\frac{K}{R^{3}}\left(1-n^{2}\right) U_{r, n m} \\
N_{z z, n m} & =\frac{D}{R}\left(\nu_{(2)} U_{r, n m}\{ \pm n\} \nu_{(2)} U_{\theta, n m}\left[ \pm \gamma_{m}\right] R U_{z, n m}\right)+\frac{K}{R^{3}}\left(\gamma_{m} R\right)^{2} U_{r, n m} \\
N_{\theta z, n m} & =\frac{D}{R} \frac{1-\nu_{(2)}}{2}\left(\left[\mp \gamma_{m}\right] R U_{\theta, n m}\{\mp n\} U_{z, n m}\right) \\
& +\frac{K}{R^{3}} \frac{1-\nu_{(2)}}{2}\{\mp n\}\left(\left[\mp \gamma_{m}\right] R U_{r, n m}+U_{z, n m}\right)
\end{array}\right\}
$$




$$
\left.\begin{array}{l}
M_{\theta \theta, n m}=\frac{K}{R^{2}}\left\{1-n^{2}-\nu_{(2)}\left(\gamma_{m} R\right)^{2}\right\} U_{r, n m} \\
M_{z z, n m}=-\frac{K}{R^{2}}\left[\left\{\left(\gamma_{m} R\right)^{2}+\nu_{(2)} n^{2}\right\} U_{r, n m}+\nu_{(2)}\{ \pm n\} U_{\theta, n m}+\left[ \pm \gamma_{m}\right] R U_{z, n m}\right] \\
M_{\theta z, n m}=\frac{K}{R^{2}}\left(1-\nu_{(2)}\right)\left(\{\mp n\}\left[\mp \gamma_{m}\right] R U_{r, n m}-\frac{\left[\mp \gamma_{m}\right] R}{2} U_{\theta, n m}+\frac{\{\mp n\}}{2} U_{z, n m}\right)
\end{array}\right\} .
$$

\section{References}

Amorosi, A., Boldini, D., 2009. Numerical modelling of the transverse dynamic behaviour of circular tunnels in clayey soils. Soil Dynamics and Earthquake Engineering 29, 1059-1072.

Asakura, T., Sato, Y., 1996. Damage to mountain tunnels in hazard area. Soils and foundations, 301-310.

Chen, W., Shih, B., Chen, Y., Hung, J., Hwang, H., 2002. Seismic response of natural gas and water pipelines in the ji-ji earthquake. Soil Dynamics and Earthquake Engineering 22, 1209-1214.

Flügge, W., 1973. Stress in Shells. Berlin and New York: Springer-Verlag.

Hashash, Y., Hook, J., Schmidt, B., et al., 2001. Seismic design and analysis of underground structures. Tunnelling and Underground Space Technology 16, 247-293.

Li, T., 2012. Damage to mountain tunnels related to the wenchuan earthquake and some suggestions for aseismic tunnel construction. Bulletin of Engineering Geology and the Environment 71, 297-308. 
Meguid, M., Dang, H., 2009. The effect of erosion voids on existing tunnel lining. Tunnelling and underground space technology 24, 278-286.

Meguid, M.A., Kamel, S., 2014. A three-dimensional analysis of the effects of erosion voids on rigid pipes. Tunnelling and underground space technology 43, 276-289.

Mow, C., Pao, Y., 1971. The Diffraction of Elastic Waves and Dynamic Stress Concentrations. Rand Corporation.

Park, K., Tantayopin, K., Tontavanich, B., Owatsiriwong, A., 2009. Analytical solution for seismic-induced ovaling of circular tunnel lining under no-slip interface conditions: A revisit. Tunnelling and Underground Space Technology 24, 231-235.

Tianbin, L., 2008. Failure characteristics and influence factor analysis of mountain tunnels at epicenter zones of great wenchuan earthquake. Journal of Engineering Geology 16, 742-750.

Wang, J., Huang, H., Xie, X., Bobet, A., 2014. Void-induced liner deformation and stress redistribution. Tunnelling and underground space technology $40,263-276$.

Wang, W., Wang, T., Su, J., Lin, C., Seng, C., Huang, T., 2001. Assessment of damage in mountain tunnels due to the taiwan chi-chi earthquake. Tunnelling and underground space technology 16, 133-150.

White, R., 1958. Elastic wave scattering at a cylindrical discontinuity in a solid. The Journal of the Acoustical Society of America 30, 771-785. 
Wolf, J.P., 1985. Dynamic soil-structure interaction. Prentice-Hall Englewood Cliffs, New Jersey.

Yashiro, K., Kojima, Y., Shimizu, M., 2007. Historical earthquake damage to tunnels in japan and case studies of railway tunnels in the 2004 niigatakenchuetsu earthquake. Quarterly Report of RTRI 48, 136-141.

Yasuda, N., Tsukada, K., Asakura, T., 2017. Elastic solutions for circular tunnel with void behind lining. Tunnelling and Underground Space Technology 70, $274-285$.

Yeh, G., 1974. Seismic analysis of slender buried beams. Bulletin of the Seismological Society of America 64, 1551-1562.

Zhang, X., Jiang, Y., Sugimoto, S., 2018. Seismic damage assessment of mountain tunnel: A case study on the tawarayama tunnel due to the 2016 kumamoto earthquake. Tunnelling and Underground Space Technology $71,138-148$. 
Table C.1: Material properties.

\begin{tabular}{lcc}
\hline Parameters & Ground & Lining \\
\hline Young's modulus (GPa) & 0.30 & 30 \\
Poisson's ratio & 0.30 & 0.20 \\
Density $\left(\mathrm{kg} / \mathrm{m}^{3}\right)$ & 2000 & 2300 \\
Radius $(\mathrm{m})$ & & 5.0 \\
Lining thickness $(\mathrm{m})$ & & 0.50 \\
\hline
\end{tabular}
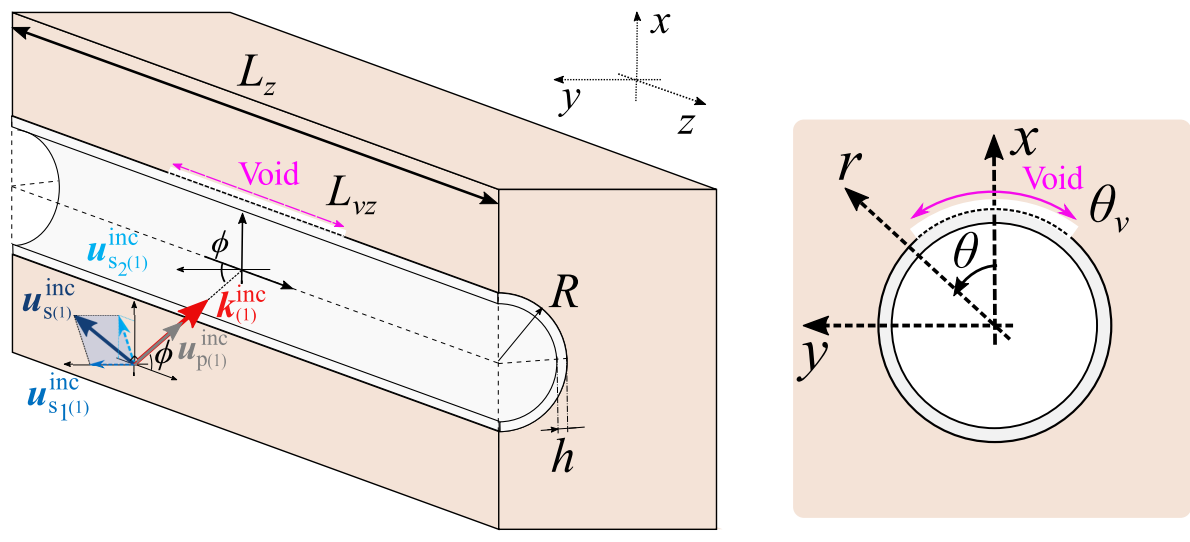

Figure C.1: A cylindrical tunnel with a void behind the lining subjected to an obliquely incident plane harmonic seismic wave. 


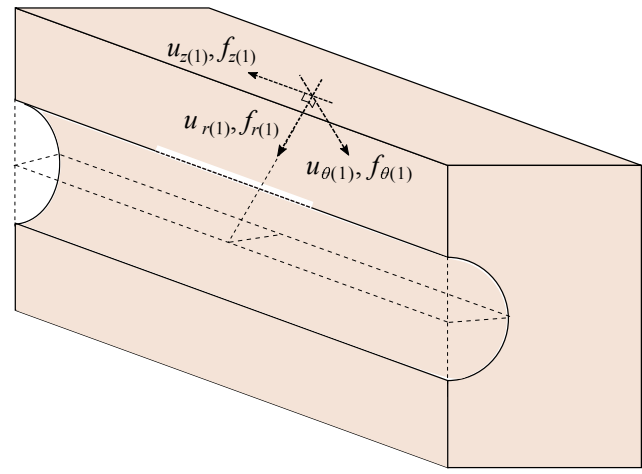

(a)

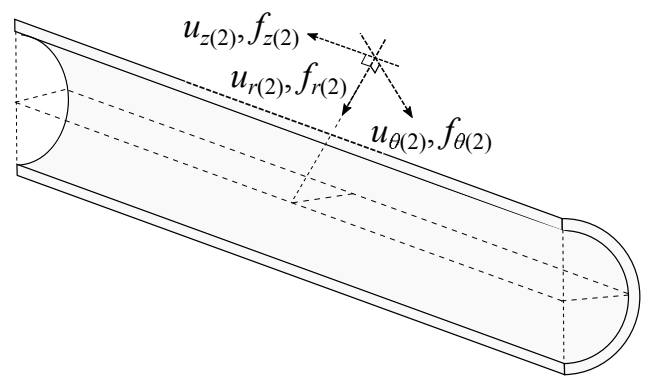

(b)

Figure C.2: Substructures to evaluate the displacement and traction under the groundlining interaction: (a) the ground system and (b) the lining system.

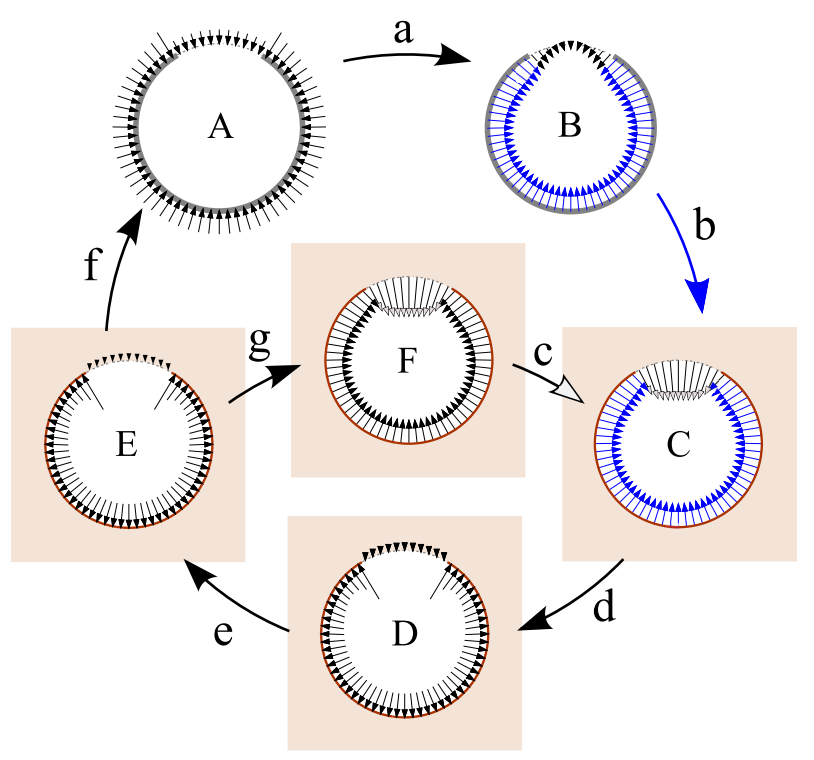

Figure C.3: Illustration of the calculation procedure to determine the solution. 


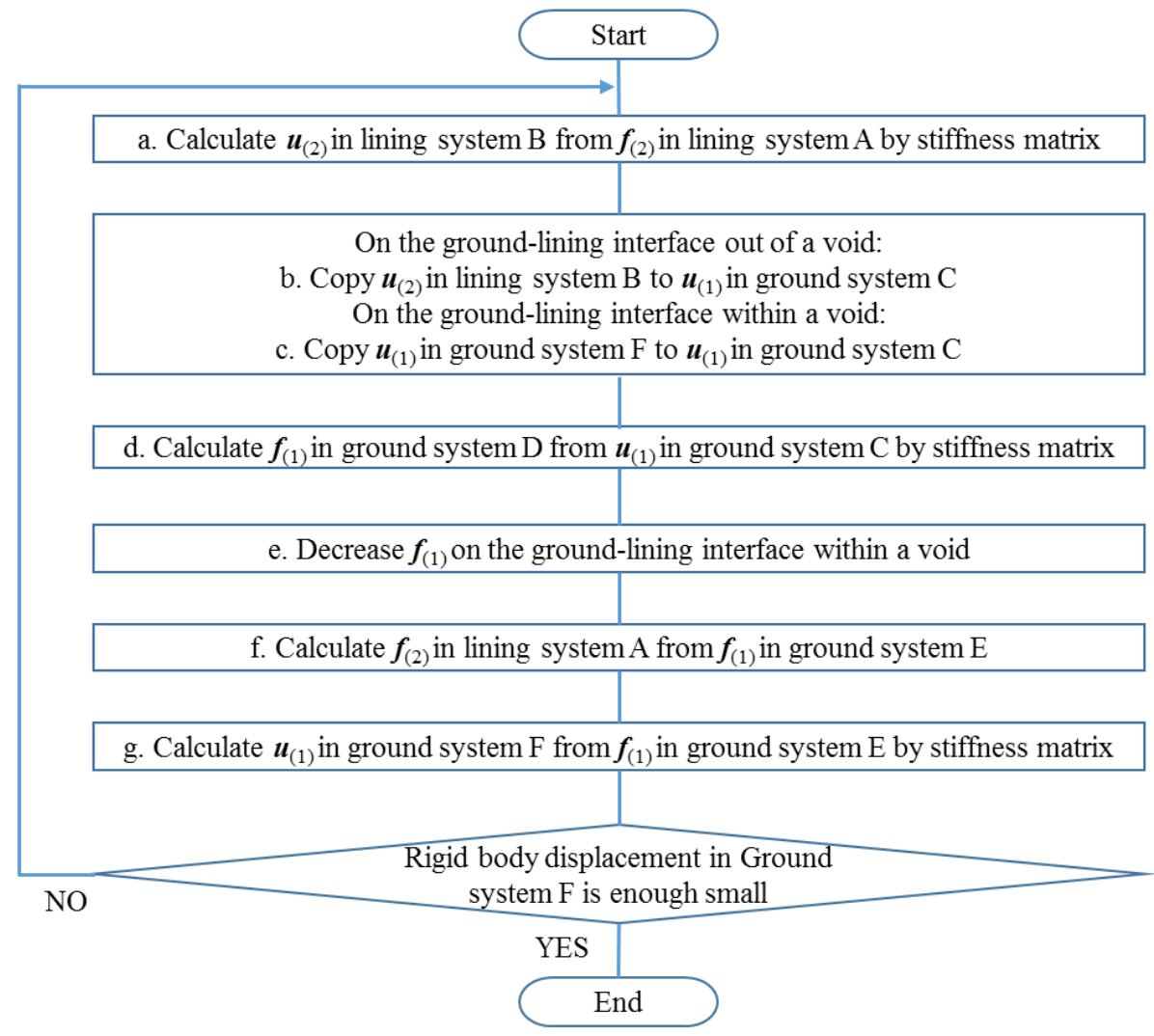

Figure C.4: Flowchart of the calculation procedure. 


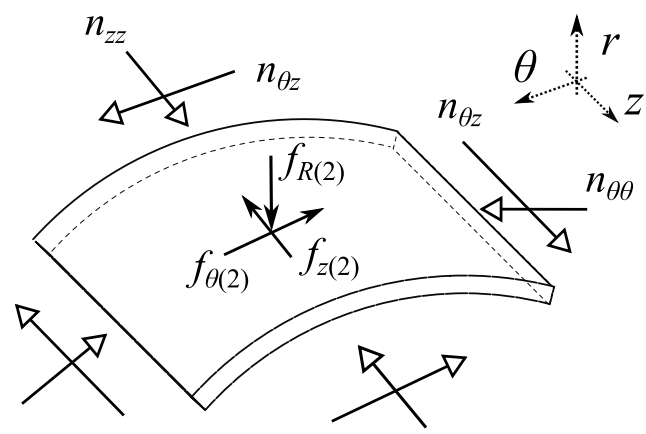

(a)

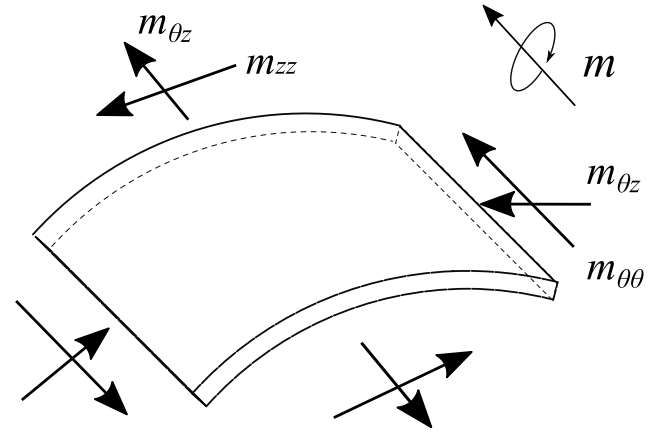

(b)

Figure C.5: Sign convention of the shell element: (a) thrust and (b) moment. 


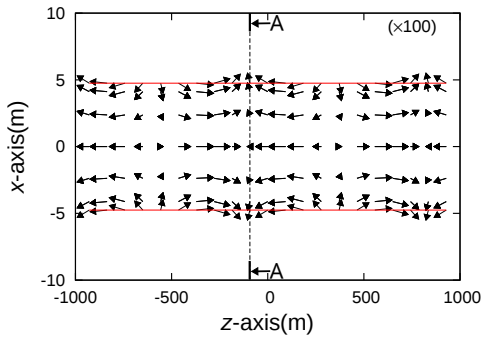

(a)

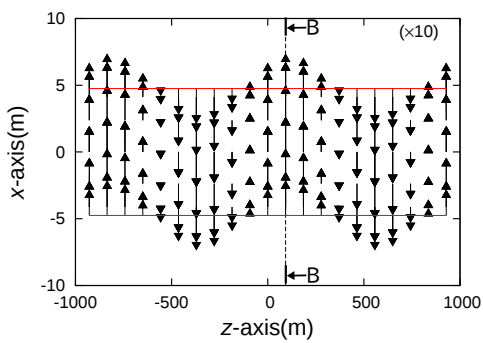

(c)

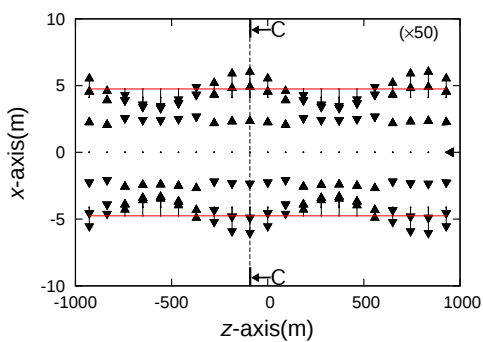

(e)

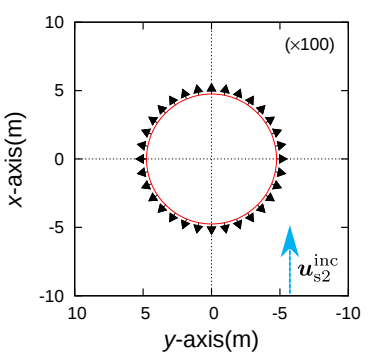

(b)

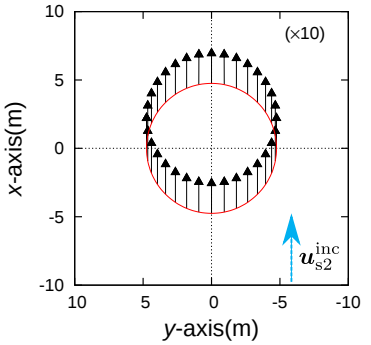

(d)

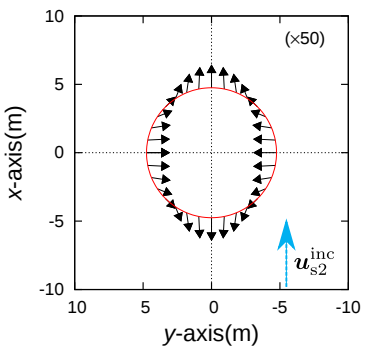

(f)

Figure C.6: Characteristic deformation modes of the lining under $\mathrm{S}_{2}$-wave incidence $(f=$ $1.0 \mathrm{~Hz}$ and $\phi=75^{\circ}$ ): (a) the zeroth mode deformation in the longitudinal section, (b) the zeroth deformation in the cross-section AA, (c) the first mode deformation in the longitudinal section, (d) the first mode deformation in the cross-section BB, (e) the second mode deformation in the longitudinal section, and (f) the second mode deformation in the cross-section CC. 


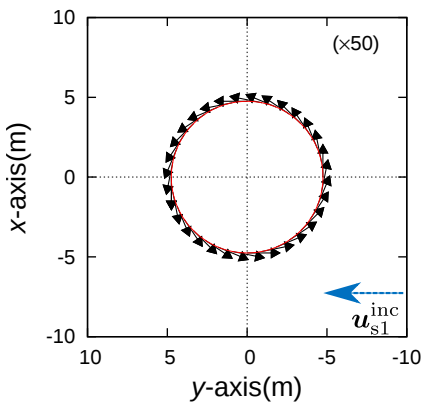

(a)

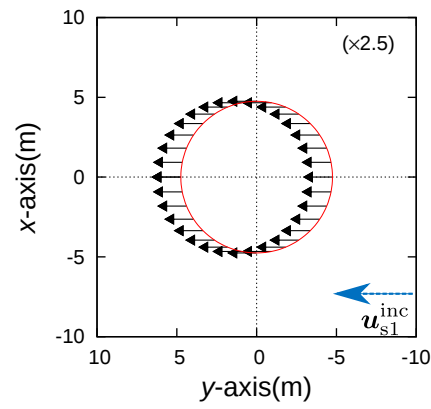

(b)

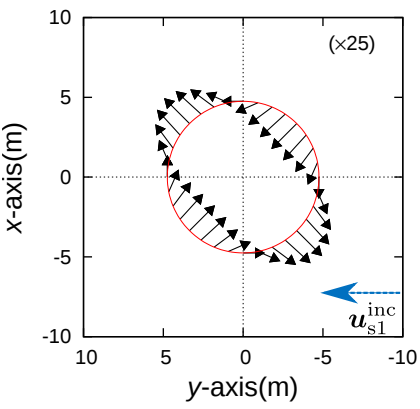

(c)

Figure C.7: Characteristic deformation modes of the lining in the cross-section under $\mathrm{S}_{1}$ wave incidence $\left(f=1.0 \mathrm{~Hz}\right.$ and $\left.\phi=75^{\circ}\right)$ : (a) the zeroth mode deformation, (b) the first mode deformation, and (c) the second mode deformation.

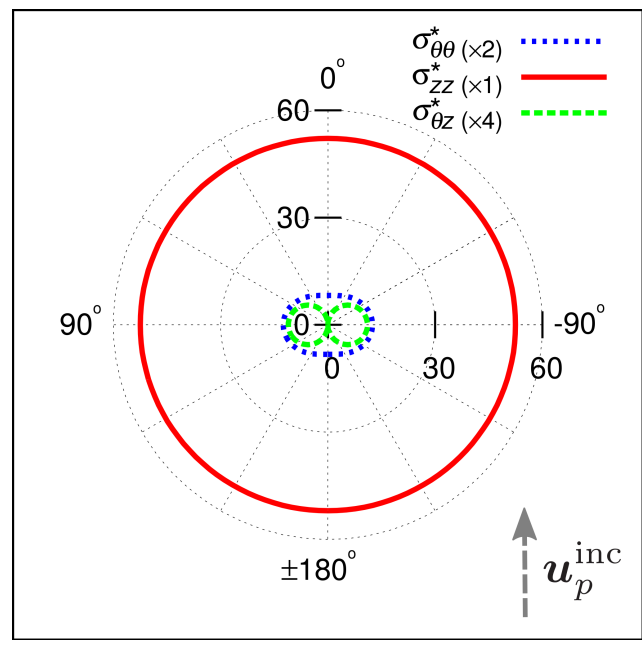

(a)

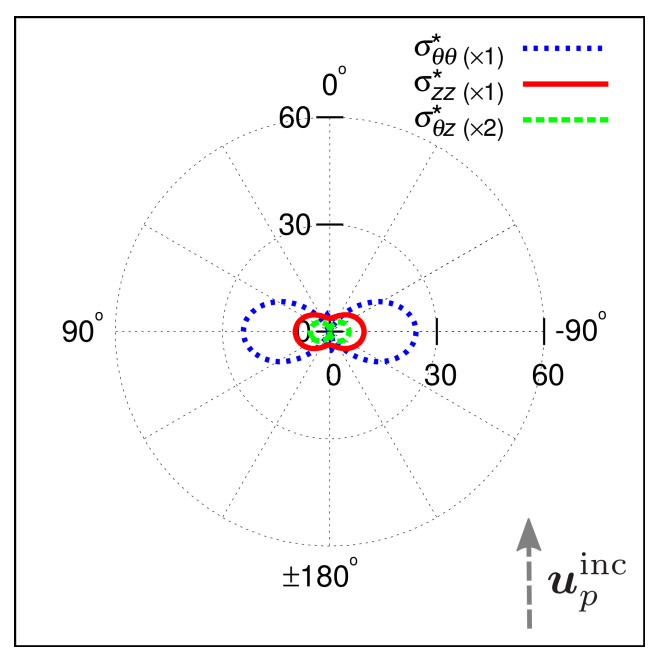

(b)

Figure C.8: Maximum stress distribution of the inner surface of the lining under P-wave incidence $(f=1.0 \mathrm{~Hz})$ : (a) $\phi=15^{\circ}$ and (b) $\phi=75^{\circ}$. 


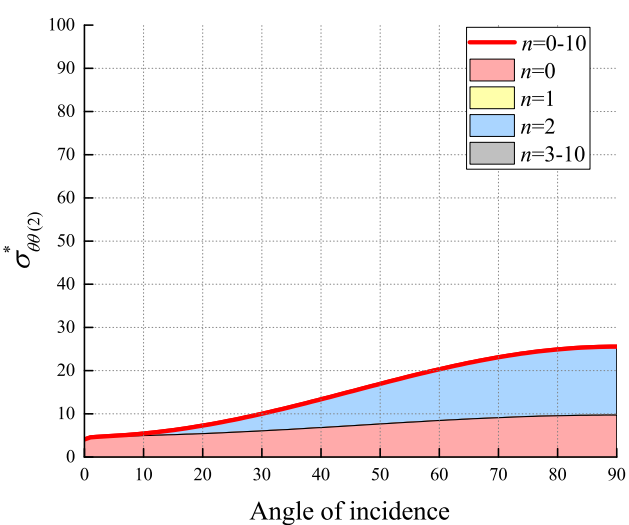

(a)

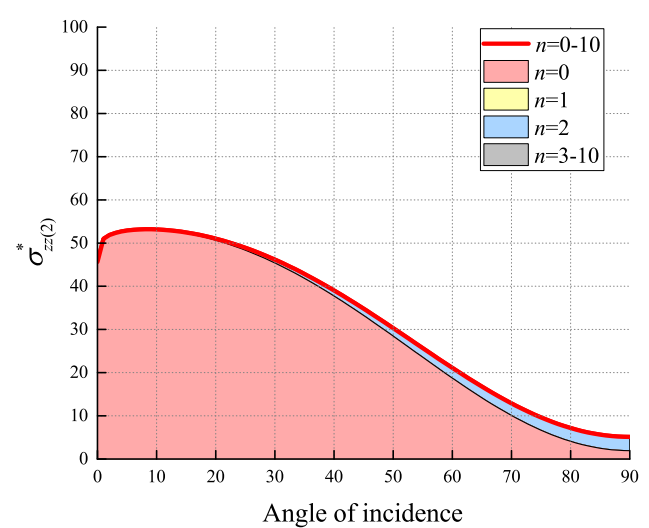

(b)

Figure C.9: Maximum stress of the inner surface of the lining at $\theta=90^{\circ}$ under P-wave incidence and the contribution of each mode $(f=1.0 \mathrm{~Hz})$ : (a) relationship between $\phi$ and $\sigma_{\theta \theta}$, and (b) relationship between $\phi$ and $\sigma_{z z}$. 


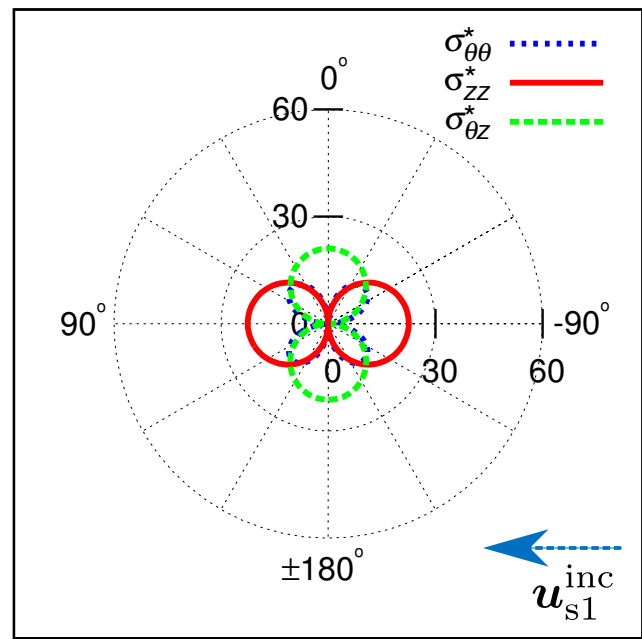

(a)

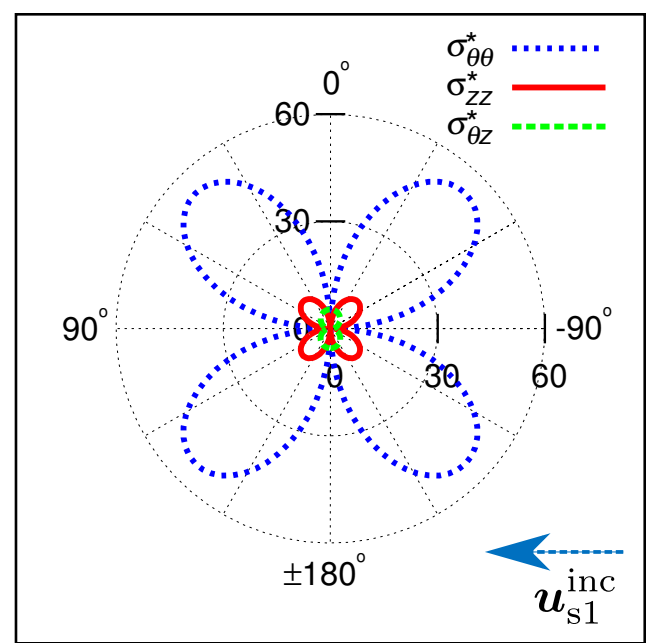

(b)

Figure C.10: Maximum stress distribution of the inner surface of the lining under $\mathrm{S}_{1}$-wave incidence $\left(f=1.0 \mathrm{~Hz}\right.$ ): (a) $\phi=15^{\circ}$ and (b) $\phi=75^{\circ}$. 


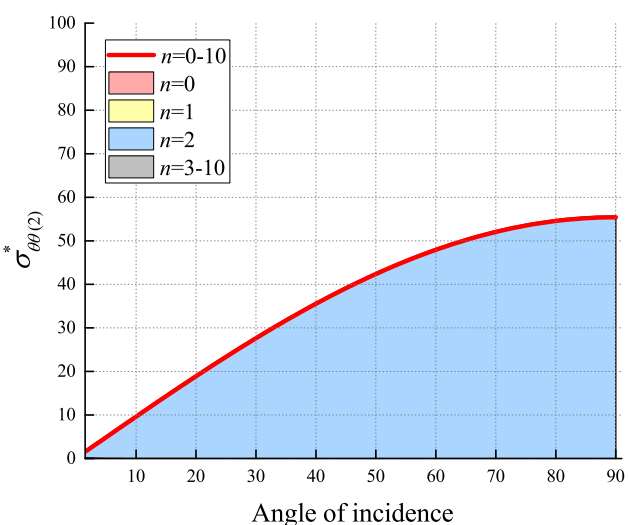

(a)

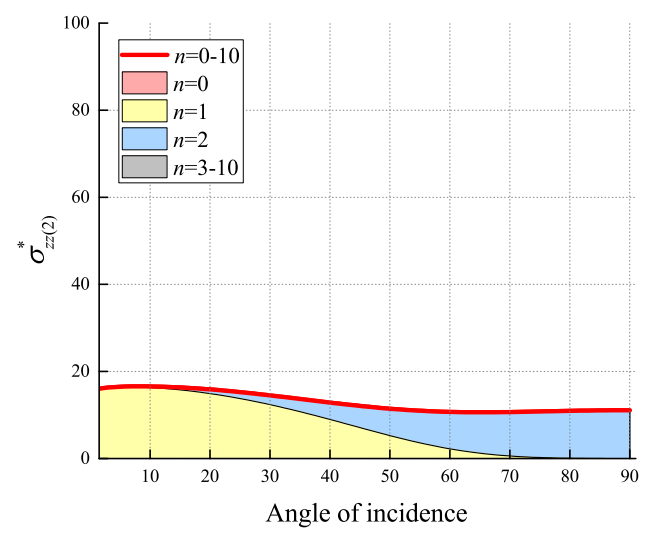

(b)

Figure C.11: Maximum stress of the inner surface of the lining at $\theta=45^{\circ}$ under $\mathrm{S}_{1}$-wave incidence and the contribution of each mode $(f=1.0 \mathrm{~Hz})$ : (a) relationship between $\phi$ and $\sigma_{\theta \theta}$, and (b) relationship between $\phi$ and $\sigma_{z z}$. 


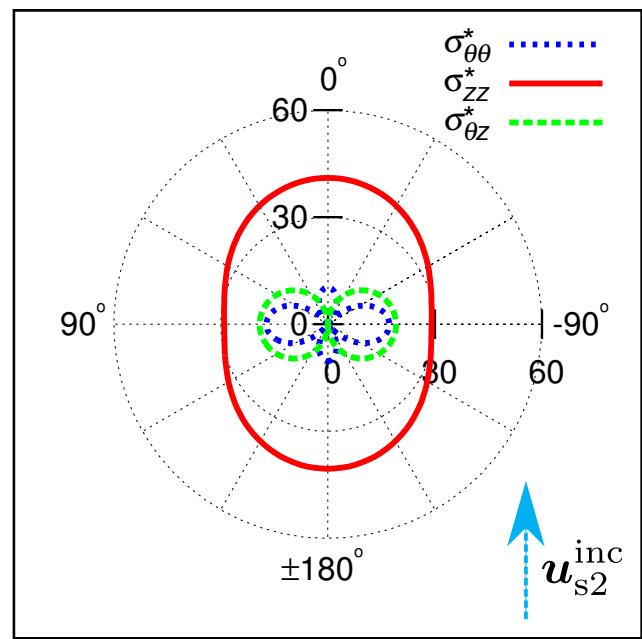

(a)

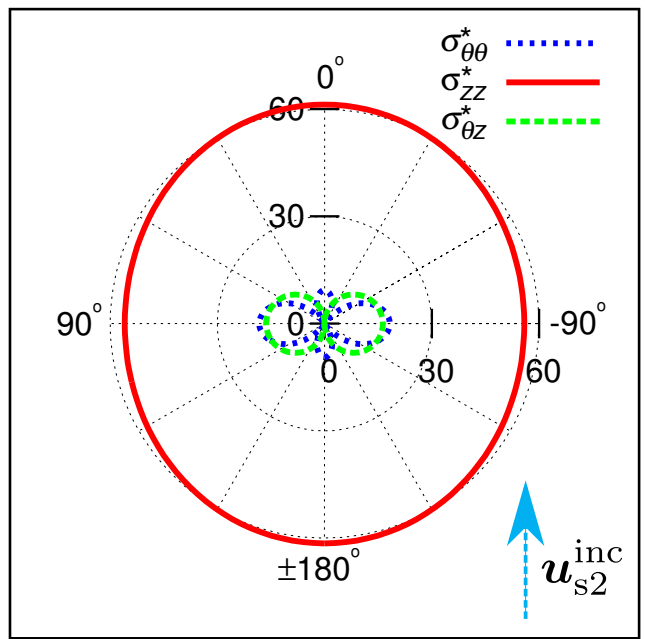

(b)

Figure C.12: Maximum stress distribution of the inner surface of the lining under $\mathrm{S}_{2}$-wave incidence $\left(f=1.0 \mathrm{~Hz}\right.$ ): (a) $\phi=15^{\circ}$ and (b) $\phi=75^{\circ}$. 


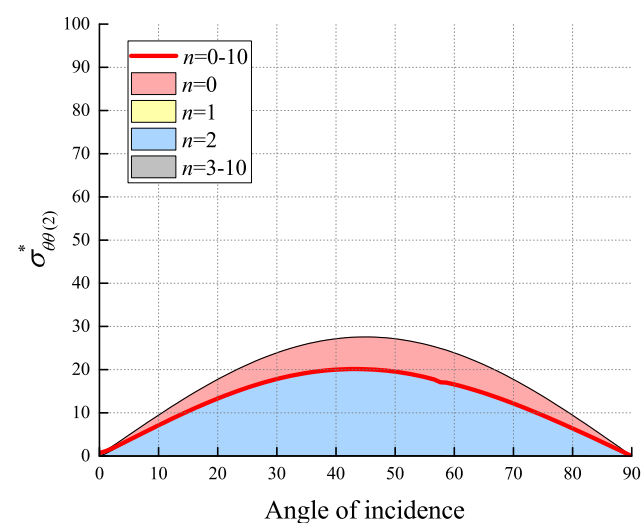

(a)

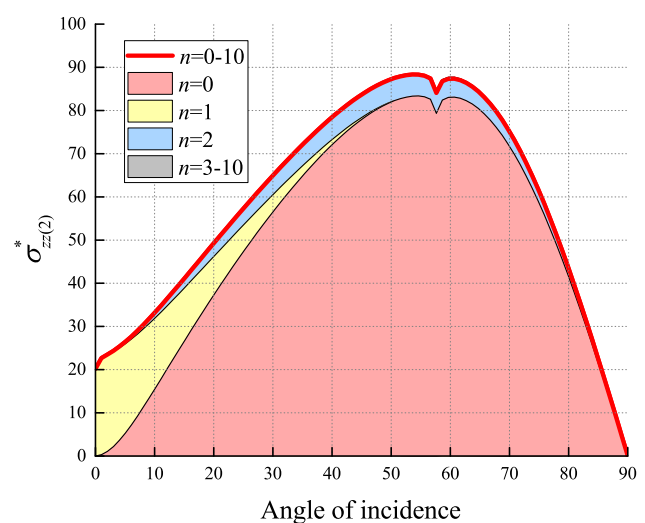

(b)

Figure C.13: Maximum stress of the inner surface of the lining at $\theta=0^{\circ}$ under $\mathrm{S}_{2}$-wave incidence and the contribution of each mode $(f=1.0 \mathrm{~Hz})$ : (a) relationship between $\phi$ and $\sigma_{\theta \theta}$, and (b) relationship between $\phi$ and $\sigma_{z z}$. 


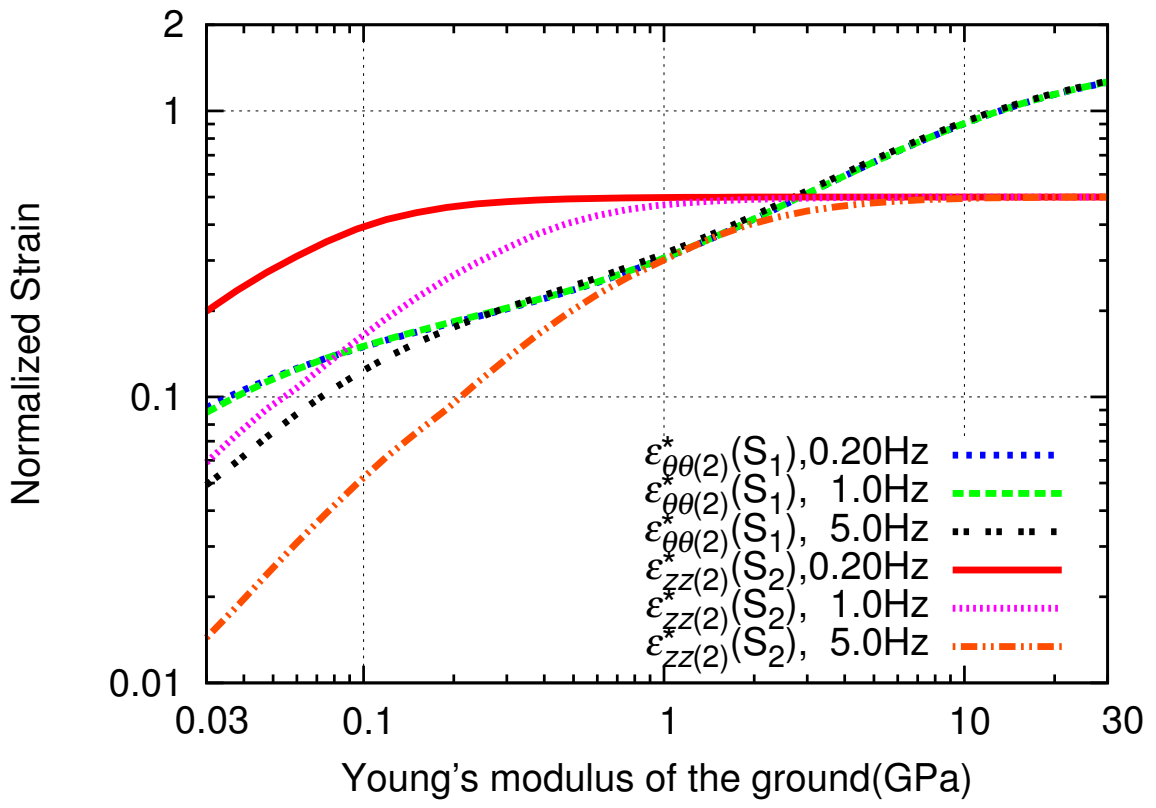

Figure C.14: Maximum $\varepsilon_{\theta \theta}^{*}$ and maximum $\varepsilon_{z z}^{*}$ on the inner surface of the lining for various Young's ratios of the ground and frequency. 


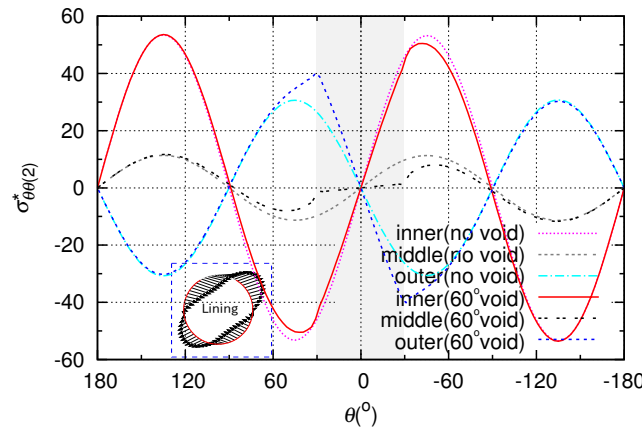

(a)

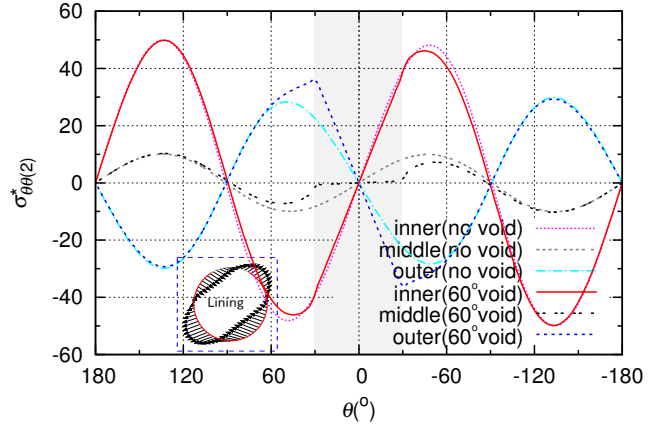

(b)

Figure C.15: Circumferential stress distributions of the inner, middle, and outer surfaces of the lining at the cross-section $z=0$ under $\mathrm{S}_{1}$-wave incidence when the maximum principal stress at the cross-section becomes maximum $\left(\phi=75^{\circ}, \theta_{v}=60^{\circ}\right.$ and $\left.L_{v z}=3 R\right)$ : (a) $f=1.0 \mathrm{~Hz}$ and (b) $f=5.0 \mathrm{~Hz}$.

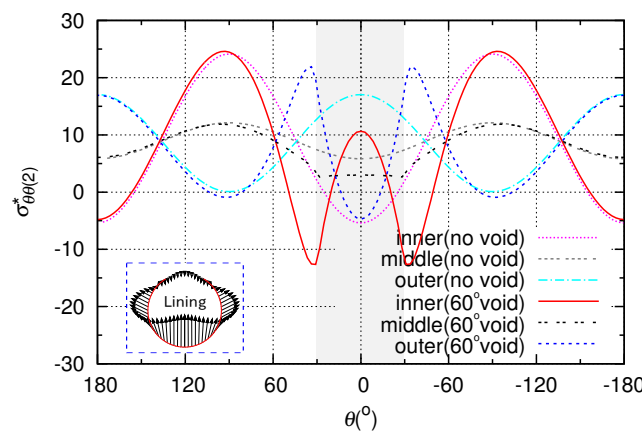

(a)

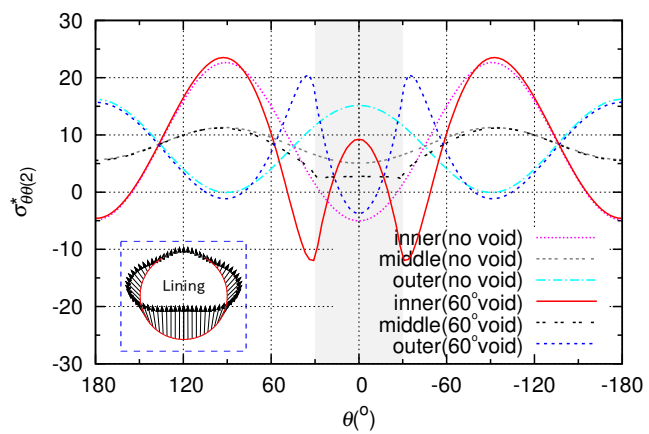

(b)

Figure C.16: Circumferential stress distributions of the inner, middle, and outer surfaces of the lining at the cross-section $z=0$ under $\mathrm{P}$-wave incidence when the maximum principal stress at the cross-section becomes maximum $\left(\phi=75^{\circ}, \theta_{v}=60^{\circ}\right.$ and $\left.L_{v z}=3 R\right)$ : (a) $f=1.0 \mathrm{~Hz}$ and (b) $f=5.0 \mathrm{~Hz}$. 


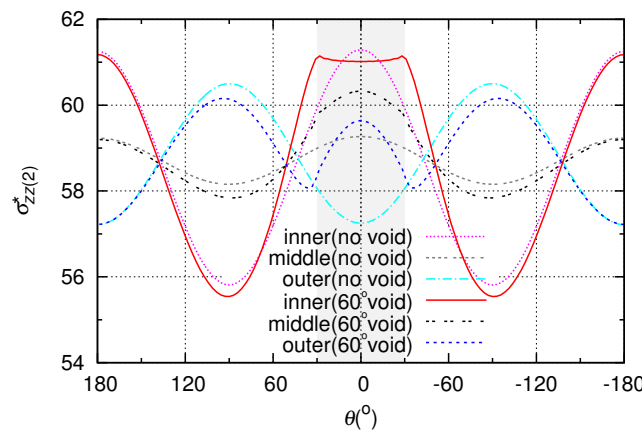

(a)

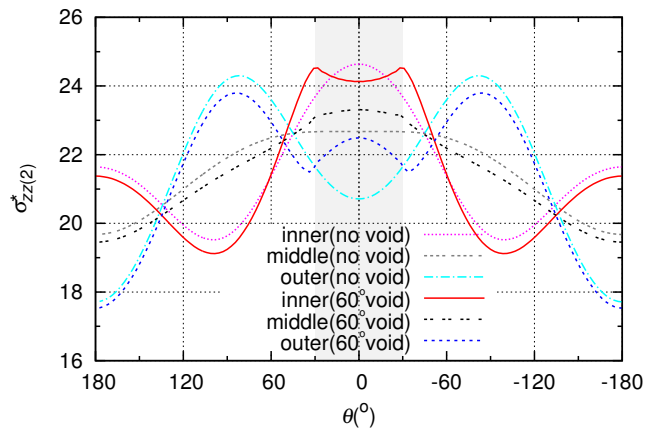

(b)

Figure C.17: Longitudinal stress distributions of the inner, middle, and outer surfaces of the lining at the cross-section $z=0$ under $\mathrm{S}_{2}$-wave incidence when the maximum principal stress at the cross-section becomes maximum $\left(\phi=75^{\circ}, \theta_{v}=60^{\circ}\right.$, and $\left.L_{v z}=3 R\right)$ : (a) $f=1.0 \mathrm{~Hz}$ and (b) $f=5.0 \mathrm{~Hz}$. 